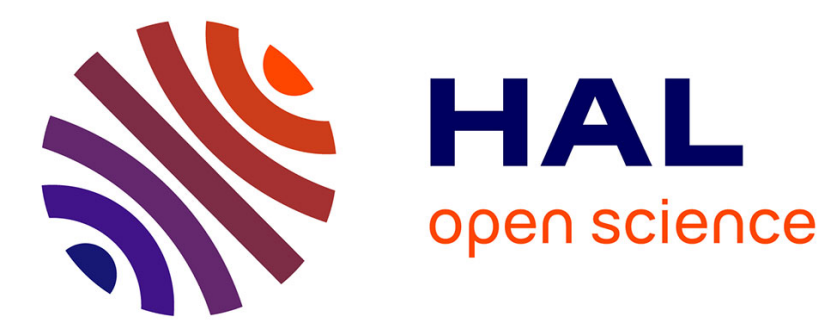

\title{
La levée des ambiguïtés syntaxiques : apport des recherches inter-langues
}

Saveria Colonna, Joël Pynte

\section{To cite this version:}

Saveria Colonna, Joël Pynte. La levée des ambiguïtés syntaxiques : apport des recherches inter-langues. Annee Psychologique, 2002, 102 (1), pp.151-187. hal-00101360

\section{HAL Id: hal-00101360 https://hal.science/hal-00101360}

Submitted on 16 May 2012

HAL is a multi-disciplinary open access archive for the deposit and dissemination of scientific research documents, whether they are published or not. The documents may come from teaching and research institutions in France or abroad, or from public or private research centers.
L'archive ouverte pluridisciplinaire HAL, est destinée au dépôt et à la diffusion de documents scientifiques de niveau recherche, publiés ou non, émanant des établissements d'enseignement et de recherche français ou étrangers, des laboratoires publics ou privés. 


\title{
LA LEVÉE DES AMBIGUİTÉS SYNTAXIQUES : APPORT DES RECHERCHES INTER-LANGUES
}

\author{
par Saveria CoLONNA ${ }^{2}$ et Joël PynTE
}

SUMMARY : Resolution of syntactic ambiguities : Evidence from crosslinguistic studies.

Determining how people convert a string of words into a representation of the structure of the sentence is a central problem in sentence processing. Studying how people handle syntactic ambiguities offers much of the information needed to tackle important issues in parsing. The first section of this article presents three types of models as a function of the solutions they propose in case of syntactic ambiguity : $1 /$ the parser forces the decision (e.g., in favour of the simplest structure) ; $2 /$ the choice is postponed until more information is available; 3 / the different structures are processed in parallel, together with other sources of information. The second section shows that these models cannot explain cross-linguistic differences observed in the resolution of syntactically ambiguous sentences. The third section presents a few new models that have been proposed to explain cross-linguistic data. This review shows how cross-linguistic findings raise major difficulties for models that have been presented so far in the literature.

Key words : syntactic ambiguity, sentence comprehension, relative clause.

\section{INTRODUCTION}

Pour les psycholinguistes, un problème central dans l'étude de la compréhension de phrases est de déterminer dans quelle mesure et comment, à partir d'une suite de mots, on construit une représentation structurée de la

1. Département de Psychologie, 29, avenue Robert-Schuman, 13621 Aixen-Provence, Cedex 1.

2. E-mail : colonna@newsup.univ-mrs.fr 
phrase. De nombreuses sources d'informations doivent être combinées lors de l'interprétation d'une phrase. Il faut utiliser l'information relative à chaque mot reconnu, ainsi que l'information contextuelle, la ponctuation, ou l'intonation, afin d'assigner une structure à la phrase. La représentation construite pour chaque phrase doit rendre compte des actions (ou états) décrits, des rôles que jouent les personnes et objets identifiés, et des relations qu'ils entretiennent entre eux. Toutes ces opérations se déroulent très rapidement. C'est parce que ces opérations sont extrêmement rapides, et parfois temporaires, qu'il est quasiment impossible de développer des mesures expérimentales capables d'appréhender ces opérations au moment où elles ont lieu. Heureusement, il est possible en utilisant des méthodes indirectes d'obtenir des informations sur la manière dont la structure d'une phrase est construite. Ces méthodes indirectes utilisent le fait que le langage est souvent ambigu. C'est le cas par exemple des phrases (1) et (2) qui comportent une ambiguïté syntaxique. En effet, dans la phrase (1), le syntagme prépositionnel (SP) « avec des jumelles » peut être attaché soit au verbe « regardait », soit au syntagme nominal (SN) « l'espion ». De même, dans la phrase (2), l'attachement de la proposition relative (PR) « qui a eu un accident » est ambigu, elle peut être attachée soit au premier nom (N1) « fille », soit au second nom (N2) « journaliste ».

(1) Le policier regardait l'espion avec des jumelles.

(2) Il connaît la fille du journaliste qui a eu un accident.

Si on présente délibérément un matériel ambigu tel que (1) et (2) aux lecteurs (ou auditeurs), on peut faire sur la base de la manière dont ils traitent l'ambiguïté des inférences sur des opérations difficilement observables directement. C'est pour cette raison que de nombreux travaux expérimentaux sur la compréhension de phrases étudient la manière dont des phrases structurellement ambiguës sont traitées. Nous allons brièvement présenter les différentes méthodes expérimentales utilisées pour étudier le traitement des ambiguïtés syntaxiques.

On distingue deux types de méthode : les méthodes dites off-line qui reflètent les processus mis en œuvre lors de la phase finale d'interprétation d'une phrase, et les méthodes en temps réel (ou on-line) qui permettent d'étudier le décours temporel des processus engagés dans la compréhension d'une phrase.

Une première méthode off-line consiste à faire passer un questionnaire au cours duquel on présente (visuellement ou auditivement) un matériel linguistique ambigu aux participants, puis on leur demande de répondre à une question de compréhension qui porte sur la région ambiguë, afin de savoir comment ils lèvent l'ambiguiité (e.g., Cuetos et Mitchell, 1988). Une seconde possibilité consiste à présenter une phrase ambiguë incomplète, puis à demander aux participants de la compléter, de nouveau afin de savoir comment ils désambiguïsent la phrase. Par exemple, dans le ques. tionnaire proposé par Zagar, Pynte et Rativeau (1997), les participants devaient lire le début d'une phrase telle que (3) et la terminer le plus spon- 
tanément possible. Dans la majorité des cas, le genre de l'adjectif utilisé pour compléter la PR permet de déterminer s'ils préferent attacher la PR à l' « avocat » ou à la « chanteuse ».

(3) Un journaliste aborda l'avocat de la chanteuse qui semblait plus...

Parmi les méthodes qui permettent d'étudier le traitement linguistique en temps réel, une première méthode consiste, à partir d'un stimulus linguistique, à demander à un participant d'effectuer une tâche de jugement le plus rapidement possible (e.g. : jugement de grammaticalité, tâche de décision lexicale), les temps de réponse alors mesurés sont supposés refléter l'importance du travail nécessaire au traitement psycholinguistique du stimulus. Dans le cas d'une tâche de jugement de grammaticalité, on prend aussi en compte la nature des réponses ainsi que le moment où la détection est effectuée. En effet, le participant peut détecter une agrammaticalité parce qu'il est engagé dans une interprétation fausse, la phrase sera alors jugée agrammaticale lorsqu'il rencontre l'information désambiguïsatrice. Cependant, la consigne de rapidité, caractéristique essentielle de ces procédures, peut induire des stratégies différentes de celles mises en jeu en situation naturelle de lecture. De plus, la présence d'une tâche ajoutée à l'activité psycholinguistique peut rendre difficile la distinction dans les effets observés entre ce qui est imputable au processus étudié et ce qui relève plus particulièrement des mécanismes mis en jeu pour l'exécution de la tâche supplémentaire.

La technique d'autoprésentation segmentée (APS) est une procédure qui permet au participant de lire à son propre rythme des textes apparaissant sur un écran (pour une description détaillée, voir Zagar, 1988). Pour commander l'apparition de segments de phrases successifs, le lecteur doit appuyer sur un bouton. L'intervalle de temps compris entre deux appuis correspond au temps d'affichage du segment sur l'écran, lequel est utilisé comme principal indicateur de l'activité de lecture. L'une des particularités de la procédure APS est de diminuer les vitesses de lecture observées en situation de lecture naturelle. Ceci est dû à la fois au fait que le défilement du texte est sous le contrôle de la motricité manuelle (plus rigide que la motricité oculaire) et au fait que la segmentation du texte empêche le lecteur d'utiliser le comportement oculaire qu'il met en ouvre habituellement. De plus, le matériel peut être présenté sous des segmentations différentes allant du mot à mot à la présentation de la phrase entière. Dans l'étude du traitement syntaxique, le choix de la segmentation peut avoir une incidence sur le cours de ce dernier en facilitant ou en inhibant le processus de segmentation naturelle. Par exemple, Gilboy et Sopena (1996) ont montré que la segmentation pouvait influencer la préférence d'attachement d'une PR ambiguë. En effet, ils observent un attachement préférentiel de la PR au premier nom ( «la hermana ») lorsque le SN complexe ( « la hermana del criado ») est présenté en un seul segment (4a), alors qu'ils n'observent pas d'attachement préférentiel lorsque le SN complexe est présenté en deux segments comme dans l'exemple (4b). 
(4a) La policia arresto a la hermana del criado / que dio a luz recientemente dos gemelos.

(La police a arrêté la sœur du servant / qui a accouché récemment de jumeaux.)

(4b) La policia arresto / a la hermana / del criado / que dio a luz recientemente dos gemelos.

La police a arrêté / la sœur / du servant / qui a accouché récemment de jumeaux.)

Enfin, le principal reproche que l'on peut adresser à la technique d'APS est que le temps d'exposition reflète de manière globale l'ensemble des activités effectuées lors de la lecture, depuis les activités de perception visuelle jusqu'aux processus d'intégration sémantique.

La technique des enregistrements des mouvements oculaires (EMO) permet de placer les participants dans une situation proche de la lecture naturelle. Cette méthode permet de séparer assez finement les différentes étapes du traitement linguistique puisqu'elle fournit plusieurs mesures, dont le temps pendant lequel le regard a fixé pour la première fois une région de l'énoncé, les endroits où et le temps pendant lequel le regard est revenu une deuxième fois sur une région déjà balayée et enfin, le temps total de fixation. L'hypothèse sous-jacente aux EMO est que la façon dont les yeux explorent le matériel reflète de manière précise le déroulement des processus cognitifs qui s'effectuent lors de la lecture. La durée des fixations est sensible aux propriétés linguistiques du texte (e.g., fréquence des mots, organisation syntaxique des mots dans la phrase, etc.). La position des régressions signale les difficultés de compréhension. En effet, elles sont souvent déclenchées quand le texte comporte des ambiguïtés syntaxiques ou référentielles.

L'analyse des potentiels évoqués consiste à enregistrer les variations de potentiels liés à des événements linguistiques. Des ondes électriques spécifiques du cerveau sont connues pour être étroitement associées à des traitements linguistiques précis. Par exemple, lorsqu'un mot imprévu survient dans une phrase, une onde négative apparaissant $400 \mathrm{~ms}$ après la lecture du

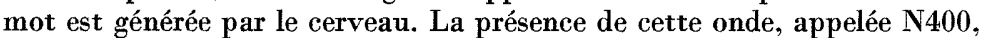
indiquerait le traitement d'une incongruité sémantique (pour une revue, voir Kutas et Van Petten, 1998). Les études concernant le traitement de l'information structurale ont permis d'identifier une onde positive centropariétale tardive (P600) et une onde négative antérieure gauche (« Left Anterior Negativity » ou LAN) corrélées avec l'analyse syntaxique d'une phrase. Lorsque le lecteur (ou l'auditeur) rencontre une ambiguïté syn. taxique, $600 \mathrm{~ms}$ après, une onde positive est générée par le cerveau. La présence de cette onde, P600, traduirait une difficulté dans le traitement syntaxique (Osterhout et Holcomb, 1993 ; Hagoort, Brown et Groothusen, 1993 ; Osterhout, 1994; Hagoort et Brown, 1994). D'autre part, une onde négative antérieure gauche (LAN) est générée par le cerveau 300 à $500 \mathrm{~ms}$ après une incongruité syntaxique (Osterhout et Holcomb, 1993 ; Coulson, 
King et Kutas, 1998 ; Hahne et Freiderici, 1999). Cette méthode permet donc d'établir une chronométrie précise des opérations mentales survenant pendant le traitement linguistique. De plus, elle présente un intérêt dans l'étude des processus impliqués dans la compréhension d'un matériel linguistique présenté auditivement. En effet, c'est la seule méthode en temps réel qui permette d'étudier les différentes étapes du traitement linguistique chez l'auditeur, de même que les EMo permettent d'étudier le déroulement des processus cognitifs chez le lecteur.

Différents modèles de la compréhension du langage ont été mis à l'épreuve dans l'étude de phrases syntaxiquement ambiguës. Ils proposent des solutions différentes en cas d'ambiguiité syntaxique locale, i.e. quand le lecteur (ou l'auditeur) se trouve dans une situation où il a le choix entre plusieurs analyses syntaxiques. En effet, les modèles de la compréhension de phrases font des hypothèses différentes quant à la stratégie adoptée en cas d'ambigü̈té syntaxique selon qu'ils adoptent une conception modulariste ou interactiviste du système cognitif. Par exemple, la solution 1 sera adoptée dans le cas d'une architecture cognitive modulaire (voir Fodor, 1983) alors que la solution 3 pourra être adoptée dans le cas d'un fonctionnement en parallèle du système cognitif.

- Solution 1 : l'ambiguïté n'est pas considérée et une seule structure est construite.

- Solution 2 : la décision est retardée jusqu'à ce qu'une information suffisante soit disponible.

- Solution 3: les différentes structures possibles sont simultanément construites en parallèle.

Notre exposé s'articulera en trois grandes parties. Dans une première partie, nous envisagerons successivement les trois solutions énoncées cidessus, ainsi que les modèles qui les sous-tendent. Nous ne développerons que les modèles les plus récents (pour une revue antérieure, voir Schelstraete, 1993), et nous limiterons aux travaux expérimentaux concernant la lecture.

Dans une seconde partie, nous verrons comment l'observation de différences inter-langues dans la structuration d'un certain type d'ambigüté syntaxique, l'attachement d'une proposition relative (PR) qui a plusieurs antécédents possibles (cf. exemple 2), a permis de mettre en évidence les limites des modèles présentés dans la première partie. Nous examinerons comment, en fonction de la solution postulée en cas d'ambiguïté syntaxique, les différents modèles de la compréhension du langage se proposent de rendre compte des différences inter-langues observées dans l'attachement d'une PR qui suit un SN complexe.

Nous consacrerons la troisième partie de notre exposé à présenter les différentes hypothèses spécifiquement proposées pour rendre compte de ces différences inter-langues. Pour chacune de ces hypothèses, nous verrons en quoi elles s'inspirent des modèles présentés précédemment. 


\section{PARTIE I : LES DIFFÉRENTES SOLUTIONS PROPOSÉES EN CAS D'AMBIGUÏTÉ SYNTAXIQUE}

\section{L'AMBIGUÏTÉ N'EST PAS CONSIDÉRÉE ET UNE SEULE STRUCTURE EST CONSTRUITE}

\subsection{La théorie du " Garden Path » de Frazier}

Selon la théorie du « Garden Path » (Frazier et Fodor, 1978; Frazier, 1979 ; Frazier et Rayner, 1982 ; Rayner, Carlson et Frazier, 1983 ; Frazier, $1987 a$ et $b, 1989,1990 a$ et $b$ ), la compréhension d'une phrase s'envisage comme le résultat final de l'activité coordonnée de différents processeurs. Chaque processeur est autonome et caractérisé par le type d'information linguistique qu'il traite. Frazier postule l'existence dans le système de compréhension du langage d'un mécanisme spécifique au traitement syntaxique, le processeur syntaxique, qui a pour unique fonction de construire la structure syntaxique de la phrase et qui, pour ce faire, utilise initialement uniquement l'information syntaxique minimale : l'ordre des mots, la catégorie syntaxique des mots (Nom, Verbe, etc.) et les règles de structuration syntaxique (cf. tableau I), i.e. l'information syntaxique qui n'est pas liée aux items spécifiques de la phrase.

TABLEAU I. - Exemples de règles de construction syntagmatique en français

Examples of rules of surface structure parsing in French

$$
\begin{gathered}
\mathbf{P}(\text { phrase }) \Rightarrow \mathrm{SN} \text { (syntagme nominal })+\mathrm{sV} \text { (syntagme verbal) } \\
\mathrm{SN} \Rightarrow \text { Article }+ \text { Nom } \\
\mathrm{SV} \Rightarrow \text { Verbe }+\mathrm{SN}
\end{gathered}
$$

En cas d'ambiguïté syntaxique locale, l'ambiguïté n'est pas considérée et une seule structure est construite. L'analyseur syntaxique choisit donc la solution la plus économique, puisqu'elle permet de prendre une décision immédiate et de ne poursuivre qu'une seule analyse, la charge en mémoire de travail étant ainsi minimale. Il adopte d'emblée une seule structure grâce à l'application de stratégies qui l'amène à construire la structure syntaxique la plus simple. Ces stratégies sont des procédures d'assemblage qui ont pour fonction de transformer les phrases en segments appartenant à un arbre syntagmatique grâce à des règles très générales de structuration syntagmatique. D'après Frazier, ces stratégies sont indépendantes de la grammaire d'une langue particulière et doivent donc s'appliquer dans toutes les langues. Les plus importantes sont la stratégie de l'attachement minimal et la stratégie de clôture finale. 
La stratégie de l'attachement minimal (minimal attachment) (Frazier et Fodor, 1978) consiste à construire la représentation syntaxique la plus simple en termes d'indicateurs syntagmatiques, i.e. celle qui contient le plus petit nombre de nœuds syntaxiques. Par exemple, la stratégie de l'attachement minimal sera mise en œuvre dans le traitement des énoncés $(5 a-b)$ proposés par Rayner, Carlson et Frazier (1983). Alors que l'énoncé $(5 a)$ respecte la stratégie de l'attachement minimal (le SP with binoculars s'attache au verbe saw), l'énoncé (5b), syntaxiquement plus complexe, correspond à un attachement non minimal (le SP with a revolver s'attache au SN objet the cop).

(5a) The spy saw the cop with binoculars.

(L'espion regardait le policier avec des jumelles.)

$(5 b)$ The spy saw the cop with $a$ revolver.

(L'espion regardait le policier avec un revolver.)

La stratégie de clôture finale (late closure) (Kimball, 1973 ; Frazier et Fodor, 1978) conduit à choisir, à attachement minimal égal, l'attachement le plus bas dans l'indicateur syntagmatique, i. e. à attacher les items lexicaux au dernier constituant construit, si c'est grammaticalement autorisé. Par exemple, dans l'énoncé (6), la stratégie de clôture finale conduit à attacher la proposition relative au SN qui la précède « colonel » plutôt qu'à « sœur ».

(6) Le journaliste interrogeait la sœur du colonel qui a eu un accident.

Les stratégies du traitement syntaxique ont été testées expérimentalement avec des phrases temporairement ambiguës, en mesurant les temps de traitement lorsque la phrase est désambiguïsée en faveur d'une interprétation préférée (i.e. suivant le principe d'attachement minimal ou de clôture finale) par rapport à une interprétation non préférée (Frazier, 1979 ; Frazier et Rayner 1982 ; Rayner, Carlson et Frazier, 1983). Les difficultés de traitement rencontrées sur ce dernier type de phrase mettent en évidence un effet dit « Garden Path », i.e. que la première structure syntaxique élaborée en appliquant la stratégie pertinente (attachement minimal ou clôture finale) n'est pas compatible avec la désambiguïsation, ceci conduit à une analyse erronée temporaire ou « Garden Path » (impasse). Le lecteur doit alors enclencher une réanalyse. Sur le plan comportemental, cette révision de l'analyse initiale se traduit par des temps de traitement plus élevés (fixations plus longues et régressions plus nombreuses). L'hypothèse d'autonomie du processeur syntaxique impose une certaine précision temporelle des mesures, puisqu'il s'agit de déterminer ce qui se passe très tôt dans le traitement. De ce point de vue, la technique des EMO paraît être la plus adaptée pour satisfaire cette exigence puisque, comme nous l'avons vu dans l'introduction, elle permet de distinguer les différentes étapes dans le traitement d'une phrase.

\subsection{Les modèles dits du " guidage lexical »}

À l'inverse de la théorie du «Garden Path » selon laquelle seule l'information syntaxique minimale guide la structuration initiale de l'énoncé, les modèles dits du « guidage lexical » suggèrent que les représen- 
tations lexicales associées aux mots de la phrase guident le traitement syntaxique d'une phrase (Ford, Bresnan et Kaplan, 1982 ; Pritchett, 1988 ; Konieczny, Hemforth, Scheepers et Strube, 1997). Selon ces modèles, le traitement syntaxique de la phrase dépend de l'information lexicale récupérée à chaque fois qu'un mot de la phrase est traité. La représentation lexicale d'un mot contient toutes les informations linguistiques concernant ce mot, notamment des informations syntaxiques, thématiques et sémantiques, de nature combinatoire qui renseignent sur les relations structurales et non structurales que le mot peut entretenir avec d'autres mots. Ces informations de nature combinatoire auraient une influence primordiale sur les opérations de structuration syntaxique. De telles informations pourraient servir à interpréter immédiatement des structures syntaxiques ambiguës, en particulier l'information disponible dans le verbe. La reconnaissance d'un verbe rendrait en effet disponibles :

- l'information de sous-catégorisation associée au verbe, i.e. le type de constituants qui peut servir d'arguments au verbe (e.g., SN objet, $\mathrm{SP}$, etc.) et les arguments qu'il admet préférentiellement ou obligatoirement (e.g., un verbe intransitif n'admet aucun argument (bavarder), un verbe monotransitif admet un seul argument (apercevoir), un verbe ditransitif admet deux arguments (accuser)) ;

- et la structure thématique associée au verbe, i.e. les rôles ou modes de participation des arguments à l'événement décrit par le verbe. Par exemple, pour le verbe " cuisiner », le SN sujet aura dans une construction active le rôle d'agent et le SN objet le rôle de patient.

En accord avec les hypothèses des modèles du « guidage lexical », de nombreuses données expérimentales montrent que l'information de souscatégorisation associée au verbe intervient tôt dans le traitement (Clifton, Frazier et Connine, 1984 ; Mitchell et Holmes, 1985 ; Tanenhaus, Carlson et Trueswell, 1989 ; Shapiro, Nagel et Levine, 1993 ; Trueswell, Tanenhaus et Kello, 1993 ; Adams, Clifton et Mitchell, 1998 ; Trueswell et Kim, 1998). Ces expériences suggèrent que la structure d'arguments la plus fréquente d'un verbe guide l'analyse syntaxique initiale. La première structure construite en cas d'ambiguïté ne dépendrait donc pas de stratégies générales du traitement syntaxique, mais serait guidée par l'information lexicale. Il faut cependant noter que certaines expériences ont échoué à mettre en évidence le rôle précoce de l'information lexicale (Kennedy, Murray, Jennings et Reid, 1989 ; Mitchell, 1989 ; Ferreira et Henderson, 1990 ; Igoa, Carreiras et Meseguer, 1998). De plus, pour les défenseurs de la « Garden Path », le fait que l'information de sous-catégorisation intervienne tôt dans le traitement n'implique pas nécessairement qu'elle intervienne lors de la phase initiale de structuration de la phrase.

Pour illustrer les modèles du « guidage lexical », nous avons choisi de présenter le modèle récemment proposé par Konieczny, Hemforth, Scheepers et Strube (1997). Puis, nous présenterons une critique des modèles du " guidage lexical » développée par Kamide et Mitchell (1999). 
Selon Konieczny et al. (1997), l'analyse syntaxique initiale d'un énoncé serait guidée par l'adoption de trois principes successifs :

(1) un constituant est préférentiellement attaché à un syntagme dont la tête lexicale a déjà été rencontrée ;

(2) un constituant est préférentiellement attaché à un syntagme dont la tête lui attribue un rôle thématique ;

(3) un constituant est préférentiellement attaché au syntagme dont la tête a été rencontrée le plus récemment.

La tête d'un syntagme est l'élément central de ce syntagme, celui qui impose ses propres caractéristiques (e.g., le genre et le nombre) aux autres éléments du syntagme. Par exemple, la tête d'un SN (e.g., « le joli chat») est le nom lui-même (ici, "chat »). De même, la tête d'un syntagme verbal (sv) (e.g., « (le joli chat) aime beaucoup le lait ») est le verbe « aime ».

Pour tester leur modèle, Konieczny et al. (1997) ont réalisé deux expériences en allemand avec la méthode des EMo. Ces expériences portaient sur l'ambiguïté d'attachement d'un syntagme prépositionnel. Trois facteurs étaient manipulés : 1 / soit le verbe attendait fortement un SP comme argument, le plus souvent comme instrument (e.g., "regarder»), soit il n'attendait pas de SP comme argument (e.g., « apercevoir ») ; 2 / le contenu du SP était soit sémantiquement biaisé en faveur de l'attachement au SV, soit sémantiquement biaisé en faveur de l'attachement au $\mathrm{SN}$; et $3 /$ le verbe était soit placé après le sujet, soit en fin de phrase (SN-sujet SN-objet SP Sv). Le modèle de Konieczny et al. (1997) prédit que lorsque le verbe est placé en fin de phrase, le SP sera préférentiellement attaché au SN car la tête lexicale du SV n'a pas encore été rencontrée. Quand le verbe est placé après le sujet, l'attachement du SP dépendra de l'information thématique associée au verbe, il sera attaché au SV lorsque le verbe attend un instrument, car dans ce cas le verbe attribue le rôle thématique du SP. Si le verbe n'attend pas d'instrument, le SP sera alors attaché à la tête lexicale la plus récemment rencontrée, i.e. le SN-objet. Si le contenu sémantique du SP est incohérent avec l'attachement ainsi réalisé, alors le processeur déclenchera une réanalyse, ceci se traduira par une augmentation des temps de lecture. Les résultats obtenus vérifient les prédictions du modèle, et montrent ainsi que l'analyse d'un énoncé ambigu est déterminée par l'ordre d'apparition des têtes lexicales, ainsi que par leurs propriétés lexicales.

Kamide et Mitchell (1999) ont mis en évidence un problème des modèles du « guidage lexical » appliqués aux langues où la tête lexicale est souvent placée après ses arguments. C'est le cas par exemple en japonais, en coréen, en allemand et en néerlandais, où le verbe peut être placé en fin de phrase. Selon les modèles du « guidage lexical ", l'attachement serait mis en attente jusqu'à ce que l'analyseur rencontre la tête lexicale. Mais cette accumulation de constituants non attachés peut créer une surcharge de la mémoire de travail, le stockage d'éléments non structurés en mémoire de travail étant plus coûteux que le stockage d'éléments structurés. Kamide et Mitchell (1999) ont réalisé une expérience en japonais, grâce à la méthode APS, afin de 
montrer que l'attachement pouvait être réalisé avant que la tête lexicale ne soit lue. Ils ont utilisé des phrases qui avaient la structure suivante :

(7a) Professeur (Nom.) étudiant (Dat.) libraire (Nom.) prêta rare ancien manuscrit (Acc.) montra.

(Le professeur montra (à l'étudiant ?) le rare et ancien manuscrit que le libraire avait prêté (à l'étudiant ?))

(7b) Professeur (Nom.) étudiant (Dat.) libraire (Nom.) déchira rare ancien manuscrit (Acc.) montra.

(Le professeur montra à l'étudiant le rare et ancien manuscrit que le libraire avait déchiré.)

(7c) Professeur (Nom.) étudiant (Dat.) libraire (Nom.) prêta rare ancien manuscrit (Acc.) déchira.

(Le professeur déchira le rare et ancien manuscrit que le libraire avait prêté à l'étudiant.)

La phrase (7a) est ambiguë, car le verbe « prêter » dans la PR et le verbe « montrer » dans la proposition principale sont tous les deux ditransitifs ; le SP « à l'étudiant » a donc deux sites d'attachement possibles. Dans la phrase $(7 b)$, seul le verbe « montrer » dans la proposition principale est ditransitif ; le SP « à l'étudiant 》 ne peut donc être attaché qu'à ce dernier et c'est la stratégie de clôture initiale. De même, dans la phrase (7c), seul le verbe « prêter » dans la PR est ditransitif ; le SP « à l'étudiant » ne peut donc être attaché qu'à ce dernier et c'est la stratégie de clôture finale. Les résultats montrent que le SP ambigu est préférentiellement attaché au verbe principal (clôture initiale) avant même que celui-ci ne soit lu, les temps de lecture augmentant au niveau du verbe principal dans la condition $(7 c)$. Contrairement aux prédictions des modèles du « guidage lexical », les résultats de Kamide et Mitchell montrent que l'attachement d'un syntagme ambigu est réalisé alors que l'information lexicale associée au verbe n'est pas encore disponible.

Les modèles du « guidage lexical » ne permettent donc pas de rendre compte des résultats de Kamide et Mitchell (1999) observés en japonais. Ceci suggère que ce dernier type de modèle ne peut fournir une explication universelle de la façon dont le système humain de compréhension de phrase fonctionne. Ces modèles doivent donc être modifiés afin de pouvoir faire des prédictions différentes quant aux facteurs qui déterminent l'analyse syntaxique d'une phrase selon que l'on est dans une langue où l'ordre des mots est libre ou fixe. En effet, nous avons vu que dans des langues où comme en japonais l'ordre des mots est libre, le traitement des phrases ne peut dépendre uniquement de l'information lexicale associée au verbe.

\section{LA DÉCISION EST RETARDÉE}

JUSQU'À CE QU'UNE INFORMATION SUFFISANTE SOIT DISPONIBLE

Les modèles dits « à engagement minimal » (suivant la terminologie de Frazier et Rayner, 1982) proposent qu'en cas d'ambiguïté syntaxique, le 
traitement est temporairement suspendu jusqu'à ce que l'analyseur rencontre l'information suffisante pour résoudre l'ambiguïté (Marcus, Hindle et Fleck, 1983 ; Kennedy, Murray, Jennings et Reid, 1989 ; Marcus et Hindle, 1990 ; Perfetti, 1990, Smith, Meier et Foss, 1991 ; Weinberg, 1993 ; Sturt et Crocker, 1996). Selon ces modèles, l'intégration de la région ambiguë dans la représentation finale de la phrase est réalisée lorsque l'analyseur dispose de l'information désambiguïsatrice qui peut être de nature syntaxique ou non syntaxique. Par exemple, Perfetti (1990) propose que lors de l'analyse initiale de la phrase, les « petites » unités linguistiques comme les syntagmes nominaux et les syntagmes prépositionnels sont assemblées, mais que leur attachement aux nœuds supérieurs est laissé en suspens. L'attachement de ces «petites » unités dépendra d'informations non structurales.

Pour illustrer les modèles « à engagement minimal », nous avons choisi de présenter le modèle développé par Sturt et Crocker (1996). Ce modèle se base sur l'hypothèse proposée par Marcus dans la théorie « de la description »(Marcus, Hindle et Fleck, 1983; Marcus et Hindle, 1990) selon laquelle lors de l'analyse structurale d'une phrase, les différents syntagmes, au lieu d'être attachés, reçoivent une analyse syntaxique dite "sousspécifiée », i.e. que l'analyseur construit une description de l'arbre syntagmatique où les différents syntagmes sont liés entre eux par des relations de dominance et d'antériorité. Ce formalisme ne nécessite donc pas que toutes les relations structurales soient entièrement spécifiées à chaque étape de l'analyse. Ainsi, comme dans les autres modèles « à engagement minimal », l'analyse syntaxique est partiellement suspendue, puis mise à jour lors d'une phase ultérieure du traitement. Sturt et Crocker distinguent deux cas de figures possibles en cas d'ambiguïté syntaxique, soit l'information désambiguïsatrice ne modifie pas les relations de dominance et d'antériorité établies, soit elle les modifie, ceci se traduira alors par une augmentation du temps de traitement. Dans ce dernier cas, les prédictions du modèle sont donc les mêmes que pour la «Garden Path». Par exemple dans la phrase (8a), Sturt et Crocker prédisent une difficulté de traitement (ou effet "Garden Path ») lorsque l'analyseur rencontre la forme verbale had been drinking, car celui-ci modifie les relations de dominance et d'antériorité déjà réalisées (cf. fig. 1). Par contre dans l'exemple (8b), Sturt et Crocker ne prédisent pas de difficulté de traitement, car la forme verbale had been drinking ne modifie pas les relations de dominance et d'antériorité réalisées (cf. fig. 2).

(8a) Before the woman visited the famous doctor had been drinking quite a lot.

(Avant que la femme ait consulté le célèbre docteur avait beaucoup bu.)

(8b) The Australian woman saw the famous doctor had been drinking quite a lot.

(La femme australienne a vu que le célèbre docteur avait beaucoup bu.) 


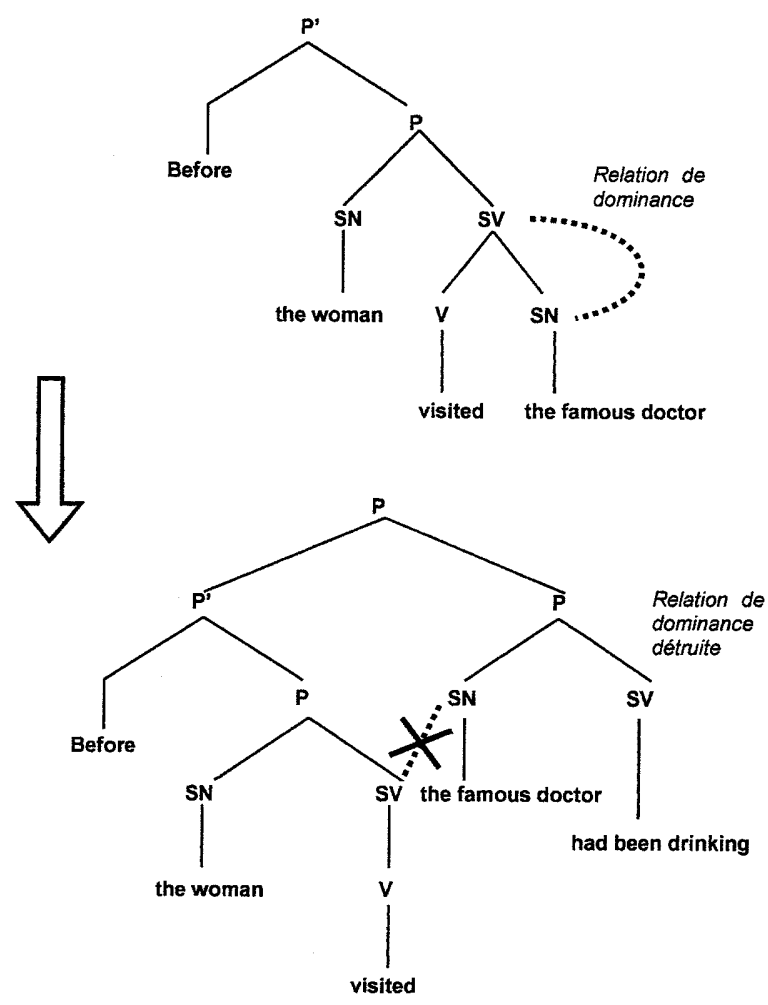

Fig. 1. - Illustration d'une réanalyse « non-monotonic » (destructrice) suivant Sturt et Crocker : d'objet du verbe visited, le $\mathrm{SN}$ ambigu the famous doctor devient le sujet de la proposition principale et de ce fait, la relation initialement établie entre le SV visited et le SN ambigu est détruite

Non monotonic update of structural relations in reanalysis of a "noun phrase / zero » ambiguity

3. LES DIFFÉRENTES STRUCTURES POSSIBLES SIMULTANÉMENT SONT CONSTRUITES EN PARALLÈLE

Selon les modèles dits « de satisfaction de contraintes » (MacDonald, Pearlmutter et Seidenberg, 1994 $a$ et $b$; Trueswell et Tanenhaus, 1994; Trueswell, Tanenhaus et Garnsey, 1994; Tabor, Juliano et Tanenhaus, 1997) en cas d'ambiguïté syntaxique, les différentes structures possibles sont simultanément construites en parallèle. Différents facteurs (ou contraintes), assignés de poids différents, permettent de renforcer une struc- 


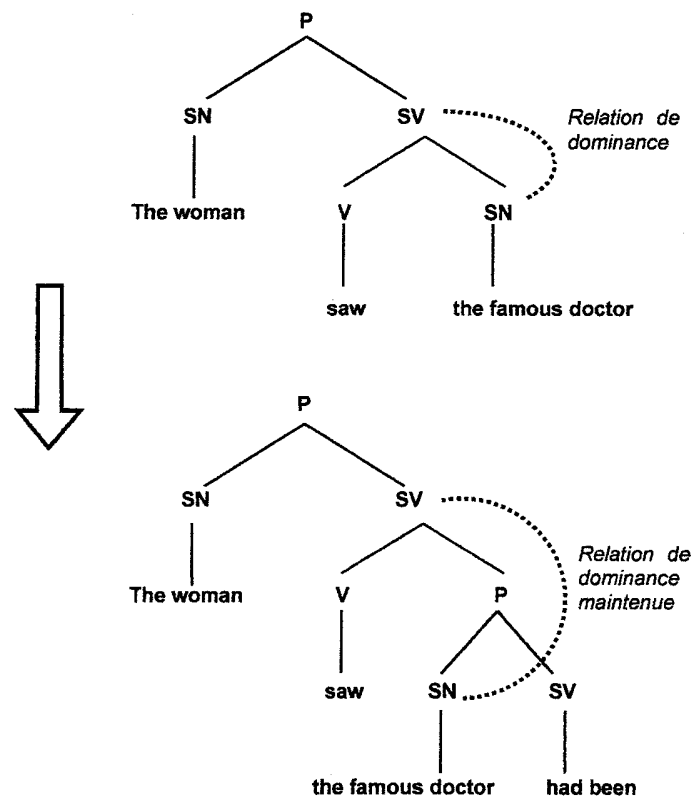

Fig. 2. - Illustration d'une réanalyse « monotonic » (non destructrice) suivant Sturt et Crocker : d'objet du verbe principal, le SN ambigu the famous doctor devient le sujet de la proposition subordonnée objet et ce, sans que la relation initialement établie entre le SV principal et le SN ambigu ne soit modifiée

Monotonic update of structural relations

in reanalysis of a " noun phrase / sentence " ambiguity

ture parmi les différentes structures possibles construites en parallèle. À chaque point de la phrase, la probabilité des différentes structures possibles est calculée. Le coût de traitement dépend de la probabilité d'occurrence de la séquence rencontrée : la séquence sera d'autant plus difficile à traiter que sa probabilité est faible. La principale caractéristique de ces modèles est que toutes les informations (fréquence d'occurrence, informations syntaxiques, sémantiques et pragmatiques) peuvent être immédiatement prises en compte pour résoudre l’ambiguïté. Pour illustrer ces modèles, nous avons choisi de présenter le modèle proposé par MacDonald, Pearlmutter et Seidenberg (1994a). Selon ce modèle dit « de satisfaction de contraintes », les ambiguïtés lexicales et syntaxiques sont gouvernées par les mêmes connaissances et mécanismes de traitement. Pour illustrer leur modèle, MacDonald et al. prennent l'exemple d'ambiguïté $(9 a-b)$ proposée par Bever (1970). 
(9a) The horse raced past the barn.

(Le cheval a galopé au-delà de l'écurie.)

(9b) The horse raced past the barn fell.

(Le cheval ayant galopé au-delà de l'écurie est tombé.)

Dans l'exemple (9a), raced est le verbe au passé de l'indicatif de la proposition principale. Dans $(9 b)$, raced est un participe passé qui introduit une proposition relative réduite (Le cheval qui avait galopé au-delà de l'écurie), et fell est le verbe de la proposition principale. Cette ambiguïté est permise en anglais car certains mots qui introduisent une proposition relative (e.g. : that had (qui avait)) peuvent être omis. Cette omission peut entraîner un problème de traitement car un verbe comme raced est ambigu, il est soit au passé de l'indicatif, soit au participe passé. Si raced est traité comme un passé de l'indicatif $(9 a)$ sera analysé correctement mais pas (9b). De même, si raced est traité comme un participe passé (9b) sera analysé correctement mais pas (9a). Selon MacDonald et al. (1994a), les deux interprétations possibles ( " proposition principale » versus « proposition relative réduite ») vont être simultanément construites en parallèle. Une de ces deux interprétations sera préférée en fonction des différentes contraintes disponibles et de leur poids respectifs. Ici, les principales contraintes qui vont déterminer le choix de l'analyseur sont, d'une part la fréquence d'occurrence de la forme raced au participe passé versus au passé de l'indicatif, et d'autre part le contexte sémantique, i.e. la plausibilité du premier nom en tant qu'agent du verbe versus en tant que patient.

Trueswell (1996) a réalisé deux expériences avec la méthode APS afin de déterminer si comme le prédisent MacDonald et al. (1994 a), la fréquence relative d'un verbe au participe passé (versus au passé de l'indicatif) peut influencer l'interprétation d'une proposition relative réduite ambiguë. Le premier verbe était soit un verbe très fréquent au participe passé, ce qui supporte l'interprétation « relative réduite », soit un verbe très fréquent au passé de l'indicatif, ce qui supporte l'interprétation "proposition principale ». Dans la première expérience, l'information sémantique supportait l'interprétation en faveur d'une relative réduite, le premier nom était tou. jours un patient plausible du verbe $(10 a)$. Au contraire, dans la seconde expérience, l'information sémantique supportait l'interprétation en faveur d'une proposition principale, le premier nom étant toujours un agent plausible du verbe $(10 b)$.

(10a) The room searched by the police had the missing weapon.

(La chambre recherchée par la police contenait l'arme manquante.)

$(10 b)$ The thief searched by the police had the missing weapon.

(Le voleur recherché par la police avait l'arme manquante.)

Dans l'expérience 1, les résultats montrent que lorsque l'information sémantique supporte l'interprétation en faveur d'une relative réduite, la difficulté de traitement disparaît lorsque le verbe est très fréquent au participe passé, mais pas lorsque c'est un verbe très fréquent au passé de 
l'indicatif. Par contre, lorsque l'information sémantique supporte l'interprétation en faveur d'une proposition principale (expérience 2), la présence d'un verbe très fréquent au participe passé ne suffit pas à éliminer les difficultés de traitement des relatives réduites. La résolution de l'ambiguïté est cependant plus rapide lorsque le verbe est très fréquent au participe passé. En accord avec les hypothèses de MacDonald et al. (1994a), ces résultats suggèrent que les ambiguïtés syntaxiques sont traitées de la même façon que les ambiguïtés lexicales. En effet, lorsque le contexte d'un mot sémantiquement ambigu supporte sa signification la plus rare, le temps de lecture augmente, de même que le temps de lecture augmente dans l'expérience 1 (contexte sémantique en faveur d'une relative réduite) lorsque le verbe est rare au participe passé.

\section{PARTIE II : APPLICATION À L'ÉTUDE DE L'ATTACHEMENT D'UNE PROPOSITION RELATIVE AMBIGÜ̈}

Comme nous l'avons vu, la théorie « Garden Path » de Frazier prédit que dans des phrases telles que (11) et (12) où un constituant ambigu a deux sites d'attachement possibles, la stratégie de clôture finale sera appliquée. Ainsi, dans l'exemple (11), yesterday sera attaché au site le plus récent died plutôt qu'à said. De même, dans l'exemple (12), la PR sera attachée à actress plutôt qu'à servant.

(11) Barbara said the politician died yesterday.

(Barbara a dit que le politicien est mort hier.)

(12) Someone shot the servant of the actress who was on the balcony.

(Quelqu'un a tiré sur la servante de l'actrice qui était au balcon.)

Cependant, dans des constructions telles que (12), les données expérimentales montrent que contrairement aux prédictions de la « Garden Path ", la stratégie de clôture finale n'est pas appliquée dans toutes les langues (cf. tableau II). Après avoir passé en revue les principales données inter-langues concernant la préférence d'attachement d'une PR qui suit un SN complexe (cf. exemple 12), nous verrons comment les différentes solutions proposées en cas d'ambiguïté syntaxique peuvent ou non rendre compte de ces différences inter-langues.

\section{DONNÉES INTER-LANGUES SUR L'ATTACHEMENT D'UNE PR QUI SUIT UN SN COMPLEXE}

Les premières données expérimentales sur la préférence d'attachement d'une PR qui suit un SN complexe « N1 de N2 » sont les travaux de Cuetos et Mitchell (1988). Ces auteurs ont fait passer un questionnaire au cours duquel des participants anglais et espagnols devaient lire dans leur langue 
Tableau II. - Données inter-langues observées quant à l'attachement d'une PR qui suit un SN complexe

Cross-linguistic data for attachment preferences concerning a relative clause preceded by a complex NP

\begin{tabular}{|c|c|c|c|}
\hline Langue & Off-line & On-line & Source \\
\hline Afrikaans & N1 & & $\begin{array}{l}\text { Mitchell, Brysbaert, } \\
\text { Grondelaers et Swanepoel } \\
(2000) \\
\end{array}$ \\
\hline Allemand & N1 & N1 (EMO) & $\begin{array}{l}\text { Hemforth, Konieczny et } \\
\text { Scheepers }(2000 \mathrm{a})\end{array}$ \\
\hline Anglais & $\begin{array}{l}\mathrm{N} 2 \\
\mathrm{~N} 2 \\
\mathrm{~N} 2\end{array}$ & $\begin{array}{c}\text { N1/N2 } \\
\text { N2 (APS/EMO) } \\
\text { N1 (APS)/N2 (EMO) } \\
\text { N2 (EMO) }\end{array}$ & \begin{tabular}{|c|} 
Cuetos et Mitchell (1988) \\
Carreiras et Clifton (1993) \\
Gilboy, Sopena, Clifton et \\
Frazier (1995) \\
Frazier et Clifton (1996, pp \\
$79-80)$ \\
Henstra (1996) \\
Carreiras et Clifton (1999) \\
\end{tabular} \\
\hline Espagnol & N1 & $\begin{array}{l}\text { N1 (APS) } \\
\text { N1 (APS) } \\
\text { N1 (APS) } \\
\text { N1 (EMO) }\end{array}$ & $\begin{array}{c}\text { Cuetos et Mitchell (1988) } \\
\text { Carreiras et Clifton (1993) } \\
\text { Gibson, Pearlmutter et } \\
\text { Torrens (1999) } \\
\text { Gilboy et al. (1995) } \\
\text { Carreiras et Clifton (1999) }\end{array}$ \\
\hline Français & N1 & $\begin{array}{l}\text { N1 (EMO) } \\
\text { N1 (EMO) } \\
\text { N2 (APS) }\end{array}$ & $\begin{array}{c}\text { Zagar, Pynte et Rativeau, } \\
\text { (1997) } \\
\text { Frenck-Mestre et Pynte } \\
\text { (1999) } \\
\text { Baccino, DeVincenzi et Job } \\
\text { (1999) }\end{array}$ \\
\hline Italien & $\begin{array}{l}\mathrm{N} 1 \\
\mathrm{~N} 1\end{array}$ & $\begin{array}{l}\text { N2 (APS) } \\
\text { N2 (APS) } \\
\text { N1 (EMO) }\end{array}$ & $\begin{array}{l}\text { DeVincenzi et Job (1993) } \\
\text { DeVincenzi et Job (1995) } \\
\text { Frenck-Mestre et Pynte } \\
(2000)\end{array}$ \\
\hline Japonais & N1 & & Kamide et Mitchell (1997) \\
\hline Néerlandais & $\mathrm{N} 1$ & $\begin{array}{l}\text { N1(APS/EMO) } \\
\text { N1 (APS) }\end{array}$ & $\begin{array}{c}\text { Brysbaert et Mitchell (1996) } \\
\text { Wijnen (1998) }\end{array}$ \\
\hline Norvégien & $\mathrm{N} 2$ & & $\begin{array}{c}\text { Ehrlich, Fernandez, Fodor, } \\
\text { Stenshoel et Vinereanu } \\
\text { (1999) }\end{array}$ \\
\hline Portugais & & N2 (APS) & Miyamoto (1999) \\
\hline Roumain & $\mathrm{N} 2$ & & Ehrlich et al. (1999) \\
\hline Russe & N1 & N1 & Sekerina (sous presse) \\
\hline Suédois & N2 & & Ehrlich et al. (1999) \\
\hline
\end{tabular}

respective des phrases ambiguës telles que (12a). Chaque phrase était suivie d'une question qui portait sur l'attachement de la PR. Par exemple, pour la phrase (12a), la question était « Qui était au balcon? ». Les résultats ont montré une préférence d'attachement au second nom (N2) en anglais, et une préférence d'attachement au premier nom (N1) en espagnol. De plus, Cuetos et Mitchell ont réalisé une expérience APS, au cours de laquelle des participants espagnols devaient lire des phrases telles que (12a-b).

(12a) Alguien disparo contra la criada de la actriz / que estada en al balcon / con su marido.

(Quelqu'un a tiré sur la servante de l'actrice / qui était au balcon / avec son mari.) 
(12b) Alguien disparo contra el criado de la actriz / que estada en al balcon / con su marido.

(Quelqu'un a tiré sur le servant de l'actrice / qui était au balcon / avec sa femme.)

Dans la condition contrôle (12a), la phrase est ambiguë et quel que soit l'attachement que le lecteur fait, aucune difficulté n'apparaît lors de la lecture du dernier segment. Par contre, dans la condition expérimentale (12b), le dernier segment peut uniquement être attaché au SN féminin « la actriz » (clôture finale). Si le lecteur attache initialement la proposition relative au SN masculin « el criado » (clôture initiale), alors il devra réanalyser la phrase à la lecture du dernier segment. Les résultats montrent que les participants mettent plus de temps pour lire le dernier segment dans la condition expérimentale que dans la condition contrôle. La stratégie de clôture finale n'est donc pas appliquée en espagnol, du moins dans ce type de construction.

Les résultats de Cuetos et Mitchell (1988) sont à l'origine de nombreuses études expérimentales menées dans différentes langues afin de mettre en évidence la préférence d'attachement d'une PR qui suit un SN complexe.

L'attachement préférentiel de la PR au premier nom a été répliqué en espagnol avec la méthode APS et les EMO (Carreiras et Clifton, 1993, 1999) et a été observé dans de nombreuses autres langues, à savoir en français avec la méthode des EMO (Zagar, Pynte et Rativeau, 1997), en néerlandais dans un questionnaire, avec la méthode APS et les EMo (Brysbaert et Mitchell, 1996 ; Wijnen, 1998), en afrikaans dans un questionnaire (Mitchell, Brysbaert, Grondelaers et Swanepoel, 2000), en allemand dans un questionnaire et avec la méthode des EMO (Hemforth, Konieczny et Scheepers, 2000 b), en russe dans un questionnaire (Sekerina, sous presse), et en japonais dans un questionnaire et avec la méthode APS (Kamide et Mitchell, 1997).

En anglais, Carreiras et Clifton (1993) n'ont pas observé avec la méthode APS d'attachement préférentiel de la PR, alors qu'avec la méthode des EMO, un attachement préférentiel de la PR à N2 a été observé (Frazier et Clifton, 1996 ; Carreiras et Clifton, 1999). Henstra (1996) suggèrent que les différences d'attachement observées en anglais (préférence d'attachement à $\mathrm{N} 2$ versus pas d'attachement préférentiel) sont dues à la sensibilité de la méthode expérimentale utilisée. En effet, elle présente deux expériences réalisées avec la méthode des EMO qui montrent un effet en faveur d'un attachement de la $P R$ à N2, alors qu'une troisième expérience réalisée avec la méthode APS ne révèle pas d'attachement préférentiel en faveur de $\mathrm{N} 1$ ou de N2.

L'attachement préférentiel de la PR au second nom observé en anglais a aussi été constaté dans d'autres langues, à savoir en portugais avec la méthode APS (Miyamoto, 1999), en suédois, en norvégien, et en roumain au cours de questionnaires (Ehrlich, Fernandez, Fodor, Stenshoel et Vinereanu, 1999), ainsi qu'en italien avec la méthode APS (DeVincenzi et Job, 1995). 
Cependant, alors que DeVincenzi et Job (1995) observent en italien un attachement au second nom avec la méthode APS, ils observent un attachement au premier nom au niveau des réponses aux questions de compréhension. Baccino, DeVincenzi et Job (1999) ont répliqué l'expérience en français avec la méthode APS et observent à nouveau un attachement initial de la PR au second nom. Ce dernier résultat est en contradiction avec les données en faveur d'un attachement initial à N1 observées en français (Zagar, Pynte et Rativeau, 1997; Frenck-Mestre et Pynte, 1999). Pynte et Colonna (2000) ont montré, en français, que la fréquence lexicale des noms dans le SN complexe influence l'attachement initial de la PR. En effet, la PR est initialement attachée au nom rare, qu'il soit placé en première ou en seconde position. Frenck-Mestre et Pynte (2000) observent que dans le matériel de Baccino et al. (matériel de DeVincenzi et Job (1995) traduit en français), le second nom est soit moins fréquent (pour la moitié des items), soit de même fréquence que N1. Frenck-Mestre et Pynte (2000) ont donc réalisé une nouvelle expérience EMO en italien où ils utilisent des phrases de DeVincenzi et Job (1995) ainsi que leur propre matériel. Dans les phrases de DeVincenzi et Job, ils observent un attachement initial au second nom, alors que dans leurs phrases, ils observent un attachement initial au premier nom. Ils concluent que l'attachement initial au second nom observé en français et en italien dépend du matériel utilisé par Baccino, DeVincenzi et Job. Nous verrons par la suite que le matériel expérimental utilisé par DeVincenzi et Job (1995) a fait l'objet d'autres critiques.

\section{L'AMBIgUÏTÉ N'EST PAS CONSIDÉRÉE} ET UNE SEULE STRUCTURE EST CONSTRUITE

L'observation de ces différences inter-langues est incompatible avec la théorie « Garden Path » selon laquelle la représentation syntaxique d'un énoncé est construite grâce à l'application de règles de structuration syntaxiques générales et indépendantes de la grammaire d'une langue.

Pour rendre compte de ces différences inter-langues, Fodor (1998) a proposé une hypothèse nouvelle qui néanmoins conserve l'idée qu'en cas d'ambiguïté syntaxique, l'ambiguïté n'est pas considérée et une seule structure est construite. Comme dans la théorie « Garden Path », Fodor propose que la structuration initiale d'un énoncé se fait sur la base de principes généraux de traitement. Fodor se base sur l'observation que la stratégie de clôture finale n'a été remise en cause que dans un seul type de construction : un SN complexe modifié par une PR (N1 prép. N2 PR). Lorsque l'ambiguïté réside dans l'attachement d'un SP qui a plusieurs sites d'attachement possibles (N1 prép. N2 SP), la stratégie de clôture finale semble être appliquée dans toutes les langues étudiées. En effet, l'attachement d'un SP ambigu au site le plus récent a été vérifié en italien (DeVincenzi et Job, 1995), en espagnol (Igoa, Carreiras et Meseguer, 1998), en anglais (Traxler, Pickering et Clifton, 1998) et en allemand (Hemforth, 
Konieczny, et Scheepers, $2000 \mathrm{~b}$ ). De même, dans des structures telles que « N1 prép. N2 prép. N3 PR » où la PR a trois sites possibles d'attachement (cf. exemple 15), les résultats montrent que la PR est préférentiellement attachée au site le plus récent (i.e. N3) en anglais (Gibson, Pearlmutter, Canseco-Gonzalez et Hickok, 1996), en espagnol (Gibson et al, 1996 ; Gibson, Pearlmutter et Torrens, 1999), en allemand (Hemforth, Konieczny et Scheepers, $2000 a$ ) et en néerlandais (Wijnen, Troost et Quené, 1999).

Selon Fodor, le statut spécial de la construction « N1 prép. N2 PR » est déterminé par le poids des constituants, la PR étant attachée et le SN complexe étant le site d'attachement. Les constituants lourds (PR et proposition adverbiale) seraient attachés suivant le principe de clôture initiale, les constituants faibles (adjectifs et adverbes) seraient attachés suivant le principe de clôture finale, et les constituants de poids moyens (syntagmes adjectivaux complexes, SP et syntagmes adverbiaux) seraient attachés suivant l'une ou l'autre des stratégies. Le poids des constituants est dépendant de leur longueur physique. Fodor fait l'hypothèse que la sensibilité à la longueur des constituants dans la construction « N1 prép. N2 PR » est due au découpage prosodique (packaging) de la phrase. Par conséquent, lors de l'analyse prosodique mentalement calculée pendant la lecture, les facteurs prosodiques influencent l'analyse syntaxique. L'analyseur prosodique obéit au principe « de balance » («Balance Principle ») qui requiert de trouver un constituant « frère » de sa propre taille. Selon le principe « de balance », les constituants courts sont attachés suivant le principe de clôture finale, car de cette façon ils reçoivent des constituants « frères 》 faibles, et les constituants longs sont attachés suivant le principe de clôture initiale, de cette façon ils reçoivent des constituants « frères " plus lourds. Ce principe est universel, mais les principes prosodiques peuvent varier d'une langue à une autre, ceci permet d'expliquer les différences d'attachement inter-langues observées. Par exemple, en anglais, où on observe que même une PR longue est attachée suivant le principe de clôture finale, le découpage prosodique opère de telle façon que le pronom relatif who, étant court et non accentué, est groupé avec N2 (lequel devient lourd). Ici, le découpage prosodique spécifique en anglais prévaut done sur le principe "de balance » et justifie le statut original de la construction « N1 prép. N2 PR » en anglais. Si l'analyseur syntaxique est sensible à des facteurs phonologiques comme la longueur d'un constituant et l'intonation, la préférence d'attachement peut être différente avec des constructions syntaxiques identiques. Fodor prédit qu'une proposition relative courte sera préférentiellement attachée à un constituant court (i.e. N2), alors qu'une PR longue sera attachée à un constituant long (i.e. au SN complexe « N1 de N2 »), donc à la tête du SN complexe N1. Cette prédiction a été vérifiée en français avec la méthode des EMo (Pynte et Colonna, 1998, 2000), et en anglais avec la méthode APs (Fernandez et Bradley, 1999). 


\section{LA DÉCISION EST RETARDÉE JUSQU'À CE QU'UNE INFORMATION SUFFISANTE SOIT DISPONIBLE}

Pour rendre compte des différences inter-langues observées dans l'attachement d'une PR qui suit un SN complexe, Frazier et Clifton (1996) ont proposé une nouvelle version de la théorie « Garden Path » qu'ils nomment « Construal ». La « Construal » propose qu'en cas d'ambiguïté syntaxique, la solution adoptée dépendra du type de relations qu'entretiennent entre eux les constituants syntagmatiques de la phrase : soit comme dans la théorie « Garden Path », l'ambiguïté n'est pas considérée et une seule structure est construite, soit comme dans les modèles « à engagement minimal », la décision est retardée jusqu'à ce qu'une information suffisante soit disponible. Dans ce dernier cas, la « Construal » reprend les hypothèses du modèle « à engagement minimal » proposé par Sturt et Crocker (1996).

Frazier et Clifton (1996) distinguent deux types de relations possibles à l'intérieur d'une phrase : les relations primaires et les relations non primaires. Les syntagmes primaires sont constitués du verbe et de ses arguments, et les syntagmes non primaires sont tous les syntagmes qui ne peuvent pas être pris pour des syntagmes primaires (e.g., les propositions relatives et les prédicats adjoints). Les syntagmes primaires sont structurés suivant les principes de la « Garden Path », i.e. qu'ils sont attachés suivant le principe de clôture finale ou d'attachement minimal, et ce, contrairement aux syntagmes non primaires qui sont initialement « associés » à un domaine thématique, puis attachés en fonction de facteurs syntaxiques et non syntaxiques. Le domaine thématique (ou domaine thêta) correspond à la projection maximale du dernier marqueur du rôle thématique. Le rôle thématique (ou rôle thêta) désigne le rôle joué par un argument vis-à-vis de son prédicat. Dans le cas des syntagmes non primaires, la « Construal » fait les mêmes prédictions que le modèle de Sturt et Crocker (1996), à savoir qu'en cas d'ambiguïté syntaxique, l'attachement est suspendu en attendant l'information suffisante. Ceci a pour principale conséquence que, contrairement aux prédictions de la « Garden Path », l'attachement d'un syntagme non primaire peut dépendre d'informations sémantiques ou pragmatiques.

Si la stratégie de clôture finale a été remise en question dans des phrases où l'ambiguïté consistait à attacher une PR qui suit un SN complexe, nous avons vu qu'elle semble être appliquée lorsque l'ambiguïté réside dans l'attachement d'un SP qui a plusieurs sites d'attachement possibles. Contrairement à la préférence d'attachement d'une PR qui varie suivant la langue examinée, dans toutes les langues étudiées jusqu’à présent, les résultats montrent qu'un SP ambigu est attaché suivant le principe de clôture finale (DeVincenzi et Job, 1995 ; Igoa, Carreiras et Meseguer, 1998 ; Traxler, Pickering et Clifton, 1998 ; Hemforth, Konieczny, et Scheepers, 2000 b). La « Construal » permet de rendre compte de ces différences obser- 
vées dans le traitement des SP et des PR. Les propositions relatives sont des syntagmes non primaires, elles ne sont donc pas attachées suivant le principe de clôture finale mais, associées au domaine thématique, puis attachées en fonction de facteurs structuraux et non structuraux, au contraire des SP qui sont des syntagmes primaires, et qui de ce fait, sont attachés suivant le principe de clôture finale.

En ce qui concerne les différences inter-langues observées dans l'attachement d'une PR qui suit un SN complexe, la « Construal » propose que plusieurs facteurs structuraux et non structuraux déterminent l'attachement d'une PR. Frazier et Clifton (1996) décrivent trois principaux facteurs que nous allons examiner ; ces facteurs sont : la structure thématique du SN complexe « N1 prép. N2 », le statut référentiel du site d'attachement de la proposition relative et le rôle des règles de conversation.

\section{La structure thématique du SN complexe}

D'après l'hypothèse de la « Construal », une proposition relative est initialement associée à la projection maximale du dernier marqueur du rôle thématique. Si l'on représente la structure d'un énoncé sous la forme d'un arbre syntagmatique, la projection maximale du marqueur du rôle thématique est le nœud au-dessus de l'élément qui assigne les rôles sémantiques aux éléments subordonnés (cf. fig. 3).

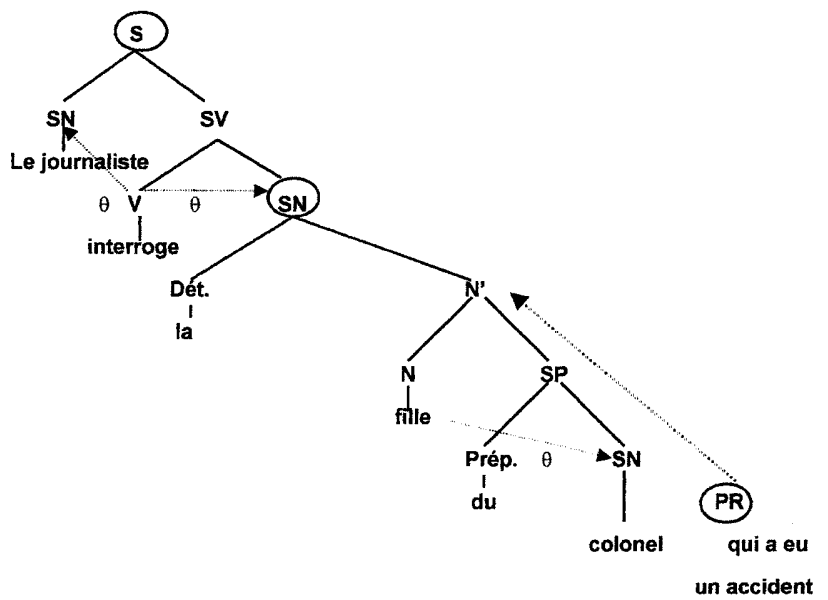

Fig. 3. - Représentation du principe « Construal » selon lequel une PR ambiguë est associée à la projection maximale du dernier marqueur du rôle $\theta$ (les nœeuds encerclés symbolisent la projection maximale d'un marqueur du rôle $\theta$ )

Phrase structure tree for a case of postmodifier attachment. Circles nodes represent thematic assigners 
L'interprétation de la proposition relative dépendra donc de la structure thématique du SN complexe, laquelle varie en fonction du statut des arguments dans le syntagme prépositionnel. Un syntagme prépositionnel est considéré comme un argument du nom si son rôle thématique est déterminé par ce nom. Si N2 est un argument de N1 (e.g., "l'image de la fille ») alors $\mathrm{N} 1$ définit le domaine thématique et la proposition relative sera associée au SN entier (N1 de N2). Par contre, si N2 n'est pas un argument de N1 mais un argument de la préposition (e.g., le garçon avec la fille), alors leSP « avec la fille » constitue le dernier domaine thématique et la proposition relative sera associée à $\mathrm{N} 2$. La préposition « de » n'est généralement pas un marqueur du rôle thématique. Dans les syntagmes nominaux suivants : « le verre de $\mathrm{N} 2$, la maison de $\mathrm{N} 2$, la fille de $\mathrm{N} 2$ », le rôle thématique de N2 est déterminé par le premier nom (N1). Donc, un syntagme prépositionnel avec la préposition « de » est considéré comme un argument de N1. Au contraire, les prépositions « avec, sur, près de, au-dessus de » sont des marqueurs du rôle thématique. Dans les syntagmes nominaux : "le verre sur la table, la maison sur la colline, la fille sur le canapé », N2 n'est pas un argument de N1, la PR sera donc associée uniquement au second nom (N2). L'attachement de la PR dépend donc en partie de la structure thématique du SN complexe, qui varie suivant le type de préposition utilisé.

Plusieurs résultats expérimentaux vérifient l'hypothèse de la « Construal » selon laquelle le type de préposition à l'intérieur du SN complexe influence la préférence d'attachement d'une PR. Dans un questionnaire en anglais, Clifton (1988) a trouvé une différence d'attachement de la proposition relative selon que le SN complexe contenait la préposition of (de) ou la préposition with (avec). Avec la préposition of, on observe la stratégie de clôture finale dans $45 \%$ des cas contre $64 \%$ avec la préposition with. Ces résultats ont été répliqués dans un questionnaire en italien, DeVincenzi et Job (1993) observent qu'avec la préposition $d i(\mathrm{de})$, la stratégie de clôture finale est appliquée dans $49 \%$ des cas contre $70 \%$ avec la préposition con (avec). En anglais, Traxler, Pickering et Clifton (1998) ont observé avec la méthode des EMO, un attachement préférentiel de la PR au second nom lorsque le SN complexe contenait la préposition with, et pas d'attachement préférentiel lorsque le $\mathrm{SN}$ complexe contenait la préposition of.

Gilboy, Sopena, Clifton et Frazier (1995) ont réalisé un questionnaire parallèlement en anglais et en espagnol au cours duquel ils présentaient des phrases ambiguës du type « N1 prép. N2 PR » afin de tester les hypothèses de Frazier et Clifton (1996). Les participants devaient choisir si la proposition relative modifiait $\mathrm{N} 1$ ou N2. Pour tester l'influence du domaine thématique dans l'interprétation des propositions relatives, ils ont utilisé des SN qui différaient par leur structure thématique (13a et $13 b$ ). Pour les phrases du type (13a) ils observent moins de choix pour l'attachement à N2 que pour les phrases du type (13b) où le syntagme prépositionnel constitue le domaine thématique. Ce résultat vérifie le principe selon lequel une proposition relative est initialement associée au domaine thématique. 
(13a) The tourists admired the museum of the city that they visited in August.

(Les touristes ont admiré le musée de la ville qu'ils ont visité(e) en août.)

(13b) The count ordered the steak with the sauce that the chef prepares especially well.

(Le comte a commandé le steak avec la sauce que le chef prépare particulièrement bien.)

En français, Frenck-Mestre et Pynte (1999) ont montré avec la méthode des EMO que la PR est préférentiellement attachée à N1 lorsqu'elle suit un SN avec la préposition « de », et à N2 lorsqu'elle suit un SN avec la préposition « avec ». Cependant, lorsque les phrases avec la préposition "avec » étaient présentées avant les phrases avec la préposition « de », ils n'observent pas d'attachement préférentiel sur ces dernières. Ce résultat montre que l'attachement de la PR peut varier en fonction de la composition de la liste expérimentale, ceci pourrait expliquer en partie les résultats observés en italien par DeVincenzi et Job (1995). DeVincenzi et Job manipulaient le type de préposition à l'intérieur du SN, en utilisant $d i$ et con, afin d'observer un éventuel effet de la structure thématique du SN complexe sur l'attachement de la proposition relative. Contrairement aux prédictions de la «Construal », ils observent avec la méthode APS, que la PR est préférentiellement attachée à N2 quelle que soit la structure thématique du SN complexe. Ce n'est qu'au niveau des réponses aux questions de compréhension qu'ils observent un effet de la préposition di (de) versus con (avec). DeVincenzi et Job (1995) concluent que la PR est initialement attachée suivant le principe de clôture finale. Frenck-Mestre et Pynte (1999) suggèrent que l'effet observé par DeVincenzi et Job en faveur d'un attachement à N2 était conditionné par le matériel utilisé qui contenait des phrases avec la préposition « avec » lesquelles, on le sait, induisent un attachement à $\mathrm{N} 2$

Quand N1 et N2 sont tous deux disponibles comme site d'attachement de la proposition relative à l'intérieur du domaine thématique, Frazier et Clifton (1996) proposent que le statut référentiel du site d'attachement de la PR influence son attachement.

\section{Le statut référentiel du site d'attachement de la proposition relative}

Frazier et Clifton (1996) proposent le « principe de référentialité » selon lequel un modificateur comme une proposition relative cherchera préférentiellement un site d'attachement référentiel. On sait qu'une proposition relative sert généralement à identifier le référent d'un SN (Crain et Steedman, 1985 ; Clifton et Ferreira, 1989). Suivant le « principe de référentialité ", un site d'attachement est « référentiel » s'il introduit ou se réfêre à une entité du discours. Dans le cas où le site d'attachement est référentiel, il sera introduit par un déterminant. 
Afin de tester le " principe de référentialité ", Gilboy et al. (1995) ont utilisé dans leur questionnaire des phrases telles que (13c). Ils observent moins de stratégies de clôture finale pour les phrases (13c) que pour les phrases $(13 a)$, vérifiant ainsi que la proposition relative est préférentiellement associée à un SN référentiel quand N1 et N2 appartiennent tous deux au même domaine thématique. En effet, dans les phrases (13c), N2 n'est pas introduit par un déterminant et n'est donc pas considéré comme référentiel.

(13c) John asked for the glass of water that was on the table.

(John a demandé le verre d'eau qui était sur la table.)

\section{Le rôle des règles de conversation}

Frazier et Clifton (1996) suggèrent que des facteurs pragmatiques comme les maximes conversationnelles de Grice (1975) peuvent influencer l'interprétation d'une proposition relative qui suit un SN complexe. En effet, en anglais l'expression « the daughter of the colonel who... » (la fille du colonel qui...) peut s'exprimer sans ambiguïté « the colonel's daughter who... » quand la proposition relative modifie daughter. La maxime « être clair et non ambigu " implique que des interlocuteurs coopératifs choisissent la structure génitive saxonne non ambiguë si une modification de N1 est attendue. Dans leur questionnaire, Gilboy et al. (1995) observent pour les phrases (13a) plus d'attachement au second nom en anglais qu'en espagnol, où il n'existe pas de forme génitive non ambiguë. Cette observation vérifie que la maxime de Grice « éviter l'ambiguïté » influence l'interprétation d'une PR qui suit un SN complexe.

Cependant, selon l'argument de Frazier et Clifton (1996), l'attachement au second nom devrait être préféré dans toutes les langues où comme en anglais, il existe une structure non ambiguë pour exprimer le génitif. Mitchell et Brysbaert (1998) affirment que c'est le cas en néerlandais, où il existe trois formes génitives possibles : la forme normande (e.g., « de hoed van vader " ( " the hat of father ")), la forme saxonne (e.g., "vaders hoed» ( «father's hat»)), et la forme avec un pronom possessif (e.g., «vader zijn hoed» ( «father his hat»)). Dans le premier cas, l'attachement de la PR est ambigu, alors que dans les deux autres cas, la PR ne peut être attachée qu'au second nom hoed. Cependant, contrairement à l'anglais, l'attachement au premier nom est préféré en néerlandais (Brysbaert et Mitchell, 1996 ; Mitchell et Brysbaert, 1998). La présence d'une forme génitive non ambiguë dans la langue ne suffit donc pas à expliquer la préférence d'attachement. On peut toutefois noter une différence entre l'anglais et le néerlandais : en effet, alors que les formes génitives saxonne et normande sont interchangeables en anglais, elles ne le sont pas nécessairement en néerlandais. L'emploi de la forme génitive saxonne est plus restreint en néerlandais, elle n'est utilisée que dans le cas des noms propres et des relations familiales. Cependant, Mitchell et Brysbaert (1998) ont réalisé deux expériences en néerlandais (APS et EMO) où ils comparaient des phra- 
ses où la forme normande était interchangeable avec la forme saxonne, et des phrases où seule la forme normande était correcte; ils observent un attachement préférentiel à N1 quel que soit le type de phrase.

Dans une langue telle que le roumain où il n'existe pas de forme génitive non ambiguë, la « Construal » prédit un attachement au premier nom, puisque les règles de conversation ne devraient pas entrer en jeu. Cependant, Ehrlich et al. (1999) ont observé en roumain un attachement préférentiel de la PR au second nom. La « Construal » ne permet pas de rendre compte de la préférence d'attachement de la PR observée en néerlandais et dans ce dernier cas.

\section{LES DIFFÉRENTES STRUCTURES POSSIBLES}

\section{SONT CONSTRUITES SIMULTANÉMENT EN PARALLÈLE}

Selon les modèles de satisfaction de contraintes, en cas d'ambiguïté syntaxique, toutes les informations peuvent être utilisées immédiatement pour résoudre l'ambiguïté. Ce type de modèle s'est peu intéressé au problème de l'attachement d'une PR qui a plusieurs antécédents possibles et aux différences d'attachement observées dans les différentes langues étudiées. Seul le modèle de MacDonald et al. (1994, p. 697-698) propose que l'attachement d'une PR est principalement déterminé par les propriétés lexicales des noms en compétition à l'intérieur du SN nominal complexe. Par exemple dans la phrase (14), si le nom « actrice » attire plus un modificateur que le nom «servante », alors l'ambiguïté sera résolue en faveur d'un attachement au nom " actrice ». Seules les propriétés lexicales de chaque nom utilisé dans les expériences permettraient d'expliquer les différences inter-langues observées. Même dans le cas où le matériel est traduit (par exemple, de l'espagnol actrice à l'anglais actress), cela ne garantit pas que les propriétés d'attraction d'un modificateur soient maintenues équivalentes d'une langue à une autre.

(14) Someone shot the servant of the actress who was on the balcony. (Quelqu'un a tiré sur la servante de l'actrice qui était au balcon.)

Cependant, Mitchell, Cuetos, Corley et Brysbaert (1995) ont montré que la préférence d'attachement reste inchangée si l'on inverse l'ordre des noms à l'intérieur du SN complexe, ceci va à l'encontre des prédictions du modèle de MacDonald et al. 


\section{PARTIE III : SOLUTIONS MIXTES PROPOSÉES POUR EXPLIQUER L'ATTACHEMENT D'UNE PROPOSITION RELATIVE QUI SUIT UN SN COMPLEXE}

Nous avons vu que les modèles de la compréhension du langage présentés jusqu'ici ne proposent pas de solution satisfaisante dans le cas de l'ambiguïté syntaxique qui consiste à attacher une PR qui suit un SN complexe. En effet, aucun d'entre eux ne permet de rendre compte de l'ensemble des données expérimentales inter-langues observées. Plusieurs hypothèses ont été alors spécifiquement proposées pour expliquer les mécanismes d'attachement d'une PR qui suit un SN complexe. Ces hypothèses partagent certaines caractéristiques avec au moins deux des modèles que nous venons de présenter, c'est pour cette raison que nous les avons appelées solutions « mixtes », nous verrons en quoi elles s'inspirent des solutions envisagées précédemment.

\section{L'HYPothèse «TUNing EXPosure 》 DE Cuetos ET Mitchell}

Pour expliquer les différences inter-langues observées dans l'attachement d'une PR, Mitchell, Cuetos, Corley et Brysbaert (1995) proposent que la fréquence d'usage dans une langue détermine la décision de l'analyseur syntaxique. Comme dans la « Garden Path », l'ambiguïté n'est pas considérée et une seule structure est construite, mais non pas grâce à des stratégies générales $\mathrm{du}$ traitement syntaxique. Ici, c'est la fréquence avec laquelle le lecteur (ou l'auditeur) a déjà résolu l'ambiguïté dans un sens plutôt qu'un autre qui détermine la décision d'attachement d'une PR. Comme dans les modèles de satisfaction de contraintes, la fréquence d'usage influence la décision initiale de l'analyseur, à la différence qu'ici, seule la fréquence d'usage de la structure syntaxique influence la décision de l'analyseur. Contrairement aux modèles de satisfaction de contraintes selon lesquels la décision d'attachement est en partie déterminée par la fréquence lexicale des items rencontrés, ici, la décision initiale ne dépend que de la fréquence d'occurrence de la structure « N1 de N2 qui... ». Cette hypothèse a été testée au moyen d'analyses de corpus.

Conformément aux données expérimentales, Mitchell, Cuetos et Corley (1992) ont réalisé une analyse de corpus qui montrait que l'attachement de la proposition relative à N1 était plus fréquent en espagnol $(60 \%)$ qu'en anglais $(38 \%)$. De même, une analyse de corpus réalisée en français (basée sur 1384 occurrences de « N1 de N2 qui ») a révélé que la proposition relative était attachée à N1 dans $62 \%$ des cas (Baltazart et Kister, 1995).

Cependant, Brysbaert et Mitchell (1996) ont observé en néerlandais un attachement préférentiel de la proposition relative à $\mathrm{N} 1$ quelle que soit la méthode utilisée (questionnaire, APS, EMO), alors que dans une analyse de 
corpus, les mêmes auteurs (Mitchell et Brysbaert, 1998) observent que l'attachement de la proposition relative à N2 est l'attachement le plus fréquent en néerlandais. Ces résultats sont donc contraires aux prédictions de l'hypothèse « Tuning Exposure » selon laquelle seule la fréquence avec laquelle le lecteur (ou l'auditeur) a déjà résolu l'ambiguïté dans le passé détermine la préférence d'attachement initiale. Ceci suggère que des facteurs non statistiques doivent influencer l'attachement initial d'une PR.

Un problème soulevé par ce type d'étude réside dans le grain d'analyse choisi. En effet, dans toute analyse de corpus, il est important de connaitre ce qui est pertinent en termes de fréquence pour le système cognitif et donc de savoir précisément ce que l'on compte. Par exemple, dans le matériel de Cuetos et Mitchell (1988) qui ne comportait que des noms animés susceptibles d'être un site d'attachement de la PR, on peut se demander si la décision des participants d'attacher la PR à N1 a été affectée par l'occurrence des structures du type « N1 de N2 » avec des noms inanimés.

\section{L'Hypothèse de Gibson, Pearlmutter,}

\section{Canseco-Gonzalez ET HickoK}

Nous avons vu que si la stratégie de clôture finale a été remise en question dans le cas de l'attachement d'une PR qui suit un SN complexe, elle semble toutefois être appliquée dans d'autres types de structures (DeVincenzi et Job, 1995 ; Igoa et al., 1998). Gibson, Pearlmutter, CansecoGonzalez et Hickok (1996) proposent donc que la stratégie de clôture finale soit aussi appliquée dans le cas de l'attachement d'une PR, mais ils postulent l'existence d'un second facteur qu'ils nomment « proximité du prédicat» ( «Predicate Proximity»), lequel favorise l'attachement aussi proche que possible du prédicat de la phrase. Ce dernier interagirait avec la stratégie de clôture finale et par là même, masquerait ses effets. Dans le cas de l'attachement d'une PR en espagnol, le second facteur créerait une préférence d'attachement à $\mathrm{N} 1$ (clôture initiale) plus forte que la préférence d'attachement à N2 (clôture finale). Gibson et al. (1996) s'inspirent donc à la fois de la « Garden Path » et des modèles de satisfaction de contraintes. En effet, ils conservent l'idée selon laquelle le principe de clôture finale (qu'ils renomment " préférence de récence ») influence l'attachement initial d'une PR, et empruntent aux modèles de satisfaction de contraintes, l'idée selon laquelle plusieurs facteurs pourraient interagir pour déterminer l'attachement initial d'une PR.

Afin de tester cette hypothèse, Gibson et al. ont présenté (APS) en anglais et en espagnol des structures complexes où trois attachements de la PR étaient grammaticalement possibles (15).

(15) N1 prép. N2 prép. N3 PR.

The lamp near the painting of the house that was damaged in the flood.

(La lampe près de la peinture de la maison qui a été abîmée dans l'inondation.) 
Si la stratégie de la clôture initiale est appliquée, alors la PR devrait être préférentiellement attachée au premier nom lamp. Après le premier nom, le second nom painting devrait être préféré au dernier nom house (N1, N2, N3). Au contraire, si la stratégie de clôture finale est appliquée, alors la PR sera préférentiellement attachée dans cet ordre : $(\mathrm{N} 3, \mathrm{~N} 2, \mathrm{N1})$. Mais si deux facteurs sont responsables de l'attachement de la PR, d'autres ordres de préférence sont alors possibles. En particulier, si l'un des facteurs favorise la stratégie de clôture initiale et l'autre facteur la stratégie de clôture finale, les préférences observées seront alors $(\mathrm{N} 1, \mathrm{~N} 3, \mathrm{~N} 2)$ ou $(\mathrm{N} 3, \mathrm{~N} 1, \mathrm{~N} 2)$. C'est ce dernier ordre de préférence $(\mathrm{N} 3, \mathrm{~N} 1, \mathrm{~N} 2)$ qu'observent les auteurs en anglais et en espagnol. L'hypothèse selon laquelle un seul facteur détermine l'attachement de la PR ne peut pas expliquer ces résultats. Gibson et al. proposent donc que le principe de « préférence de récence ", identique au principe de clôture finale, interagit avec le facteur « proximité du prédicat ». La force de ce dernier varierait en fonction de la langue, alors que le principe de préférence de récence serait fixe. La combinaison de ces deux facteurs, l'un en faveur d'un attachement avec le site d'attachement le plus proche et l'autre en faveur d'un attachement avec le site d'attachement le plus éloigné, permettrait d'expliquer les résultats contradictoires observés en anglais et en espagnol par Cuetos et Mitchell (1988) ainsi que les résultats des auteurs. Cependant, le modèle de Gibson et al. reste imprécis quant au poids du facteur " proximité du prédicat » dans les différentes langues. Le poids de ce paramètre serait élevé quand dans une langue la distance moyenne entre le verbe et son objet est grande, et inversement. Sans analyse de corpus, les auteurs affirment qu'en anglais le poids du facteur « proximité du prédicat» est faible contrairement aux langues où l'on observe un attachement préférentiel au premier nom. Gibson et al. ne précisent pas non plus comment définir la distance verbe/objet dans une langue, ceci pose des problèmes si l'on considère les langues où l'ordre des mots est libre. Selon Gibson et al., le facteur "proximité du prédicat » est faible dans les langues où l'ordre Sujet-Verbe-Objet est rigide, comme par exemple, l'anglais, le portugais et le français. Il devrait, de plus, être plus faible en portugais et en anglais qu'en français, où il est possible d'insérer un adverbe entre le verbe et son objet. Conformément aux prédictions de Gibson et al., Miyamoto (1999) observe en portugais un attachement préférentiel de la PR au second nom. Cependant, contrairement aux prédictions du modèle de Gibson et al., Ehrlich et al. (1999) observent un attachement préférentiel de la PR au second nom en suédois, norvégien et roumain, alors que l'ordre Verbe-Objet est flexible dans ces langues.

\section{L'HYPOTHÈSE D'HEMFORTH KONIECZNY ET SCHEEPERS}

Pour rendre compte des différences inter-langues observées, Hemforth et al. (Hemforth, Konieczny, Scheepers, 2000 $a$ et $b$; Hemforth, Konieczny, Scheepers, Strube, 1998) proposent suivant le même principe 
que le modèle de Gibson et al., que deux facteurs en compétition déterminent l'attachement d'une PR. L'un de ces facteurs aurait un poids constant dans toutes les langues, alors que le poids du second facteur varierait en fonction de la langue. Selon Hemforth et al., l'attachement d'une PR serait déterminé par la combinaison de processus syntaxiques et anaphoriques. En effet, l'attachement d'un pronom relatif à son antécédent peut être envisagé comme un processus anaphorique. Les auteurs distinguent les processus syntaxiques d'attachement (attachment) qui conduisent à attacher la PR au dernier constituant construit (cf. « clôture finale " et " préférence de récence ») des processus anaphoriques de liaison (binding) dirigés par les informations liées à la représentation mentale du discours. Les processus anaphoriques conduiraient à attacher la PR au premier nom. En effet, des facteurs non structuraux comme le principe de " pertinence relative" (relativized relevance) selon lequel la PR est préférentiellement attachée à la principale assertion de la phrase (Frazier, $1990 \mathrm{~b}$; DeVincenzi et Job, 1995 ; Frazier et Clifton, 1996) ou le principe de référentialité (Frazier et Clifton, 1996 ; Gilboy et al., 1995) conduiraient à attacher la PR à l'antécédent le plus haut dans l'arbre syntagmatique. Selon Hemforth et al., le poids des processus anaphoriques varie selon la langue. Ainsi, comme en anglais le pronom relatif peut souvent être omis, le poids des processus anaphoriques serait faible dans cette langue. Ce serait donc les processus syntaxiques qui détermineraient l'attachement de la PR au second nom en anglais (même lorsque le pronom relatif est présent). Pour tester cette hypothèse, Hemforth, Konieczny, Scheepers (2000 b) ont réalisé une expérience en allemand où ils comparent l'attachement d'une PR versus l'attachement d'un SP dans des énoncés du type (16a-b). La PR (16a) est préférentiellement attachée au premier nom, alors que le SP (16b) est préférentiellement attaché au second nom, quelle que soit la méthode utilisée (questionnaire et EMO). Les mêmes résultats ont été observés en anglais (Traxler, Pickering, et Clifton, 1998). Ces résultats peuvent être interprétés dans le cadre de la « Construal » qui prédit un attachement différent dans le cas d'un SP (syntagme primaire) et dans le cas d'une PR (syntagme non primaire). Hemforth et al. proposent une interprétation différente : selon eux, l'attachement du SP au second nom est entièrement déterminé par des processus syntaxiques, alors que les processus anaphoriques déterminent l'attachement de la PR.

(16a) Die Tochter der Lehrerin die aus Deutschland kam.

(La fille du professeur qui venait d'Allemagne.)

(16b) Die Tochter der Lehrerin aus Deutschland

(La fille du professeur d'Allemagne.)

Comme Gibson et al. (1996), Hemforth, Konieczny, Scheepers (2000 a) ont réalisé des expériences avec des énoncés avec trois sites d'attachement possibles pour tester leur hypothèse. Gibson et al. ont observé la préférence d'attachement suivante (N3 > N1 > N2) en anglais et en espagnol. Hemforth et al. observent dans un questionnaire en allemand, au cours duquel 
les participants devaient donner des jugements de grammaticalité, la même préférence d'attachement $(\mathrm{N} 3>\mathrm{N} 1>\mathrm{N} 2)$.

Cependant, l'attachement au second nom observé en portugais (Miyamoto, 1999) et en roumain (Ehrlich et al., 1999) va à l'encontre des prédictions de Hemforth et al., car en portugais, comme en roumain, le pronom relatif est obligatoire.

\section{CONCLUSION GÉNÉRALE}

Un premier point que nous voulons discuter est le fait que les travaux présentés se basent sur la façon dont on traite une ambiguïté syntaxique. Lorsqu'on rencontre un matériel non ambigu où le problème qui consiste à décider quelle structure choisir ne se pose pas, la question immédiate est de savoir comment nous construisons et vérifions cette structure. Malheureusement, pour des raisons que nous avons abordées dans l'introduction, il est très difficile d'obtenir des effets mesurables avec des structures non ambiguës. C'est pour cette raison que la majorité des travaux expérimentaux sur la compréhension de phrases porte sur le traitement de structures ambiguës, ou au moins temporairement ambiguës. Néanmoins, un certain nombre des opérations dont nous avons discuté entre en jeu dans le traitement d'une phrase, qu'elle soit ambiguë ou pas. Dans de tels cas, la généralisation des résultats obtenus au traitement des phrases non ambiguës ne pose pas de problème. Même si dans l'étude de phrases syntaxiquement ambiguës, on prend le risque de mesurer des mécanismes faussés par rapport aux processus mis en jeu en temps normal, ce compromis est néanmoins nécessaire pour rendre ces opérations accessibles à l'investigation expérimentale.

Nous avons vu que les travaux menés dans d'autres langues que l'anglais ont clairement montré qu'une théorie comme la « Garden Path », selon laquelle seul le principe de clôture finale (ou principe de récence) est responsable de l'attachement d'une proposition relative, n'est pas viable. Ce sont ici les recherches inter-langues qui ont permis de montrer les limites de la théorie «Garden Path ». Si les recherches s'étaient cantonnées à l'étude de la langue anglaise, il est probable qu'aucun des problèmes actuels n'auraient été mis en évidence. Les données inter-langues ont suggéré aux psycholinguistes de nouvelles propositions afin de remplacer ou de compléter le principe de « clôture finale ». L'état actuel des travaux ne permet pas de dire si une de ces propositions pourrait rendre compte des mécanismes d'attachement d'une PR mis en œuvre dans toutes les langues étudiées. En effet, à la lumière des données expérimentales, on s'aperçoit que chacun de ces modèles fait des prédictions, soit fausses, soit incomplètes. Néanmoins, dans la plupart des cas, ces prédictions pourraient être améliorées si le modèle était plus précis et plus détaillé. L'hypothèse proposée par Fodor nécessite que les principes prosodiques qui varient d'une langue à une autre 
soient mieux définis afin de pouvoir faire des prédictions quant à la préférence d'attachement d'une PR dans de nouvelles langues. L'hypothèse de la «Construal » selon laquelle les principes de Grice peuvent influencer la préférence d'attachement d'une PR doit être revue afin de ne plus prédire indistinctement un attachement à N2 dans les toutes langues où il existe une forme génitive non ambiguë. Les modèles de « satisfaction de contraintes » doivent nécessairement accorder moins d'importance aux contraintes lexicales des sites d'attachement, et explorer d'autres contraintes potentielles. L'hypothèse « Tuning Exposure » doit être plus explicite quant au grain d'analyse qui doit être retenu. L'hypothèse de Gibson et collaborateurs doit être plus précise quant au poids du facteur « proximité du prédicat » dans les différentes langues, une fois encore, de façon à pouvoir faire des prédictions dans d'autres langues. De même, Hemforth et collaborateurs ont besoin de préciser quels sont exactement les facteurs qui vont déterminer le poids relatif des deux mécanismes responsables de l'attachement d'une PR en compétition dans une langue.

En plus de ces développements théoriques, de nouvelles données expérimentales dans des langues qui n'ont pas encore été étudiées sont nécessaires. Ainsi, les limites supplémentaires des différents modèles proposés cidessus pourront être mises en évidence.

\section{RÉSUMÉ}

Comprendre comment, à partir d'une suite de mots on construit une représentation structurée de la phrase, est capital dans l'étude de la compréhension du langage. L'étude de la levée des ambigü̈tés syntaxiques permet d'obtenir des informations sur la manière dont la structure d'une phrase est construite. La première partie de cet article est consacrée à la présentation de trois catégories de modèles en fonction de la solution qu'ils proposent en cas d'ambiguïté syntaxique : 1 / l'ambigü̈té n'est pas considêrée et une seule structure est construite ; 2 / la décision est retardée jusqu'à ce qu'une information suffisante soit disponible; 3 / les différentes structures possibles sont simultanément construites en parallèle. La seconde partie montre que ces modèles ne permettent pas de rendre compte des différences inter-langues observées dans la résolution de certaines phrases syntaxiquement ambiguës, en particulier, dans le cas de l'attachement d'une proposition relative ambiguë. Dans la troisième partie sont présentês les modèles récemment proposés pour expliquer les données interlangues. Nous verrons que les modèles actuels de la compréhension de phrases ne permettent pas de rendre compte de l'ensemble des observations interlangues. relative.

Mots-clés : ambiguitté syntaxique, compréhension de phrases, proposition 


\section{BIBLIOGRAPHIE}

Adams B. C., Clifton C., Mitchell D. C. - (1998) Lexical guidance in sentence processing ?, Psychonomic Bulletin \& Review, 5 (2), 265-270.

Baccino T., De Vincenzi M., Job R. - (1999) Cross-linguistic studies of the late closure strategy : French and Italian, in V. Lombardo et M. DeVincenzi (Edit.), Cross-linguistic perspectives on language processing, Netherlands, Kluwer Academic Press, 89-118.

Baltazart D., Kister L. - (1995) Corrélation entre détermination et sélection d'un anaphorisé dans une structure $N$ de $N$, communication présentée au séminaire « Anaphore et Référence ", Nancy, France.

Bever T. G. - (1970) The cognitive basis for linguistic structures, in J. R. Hayes (Edit.), Cognition and the development of language, New York, Wiley, 279-360.

Brysbaert M., Mitchell D. C. - (1996) Modifier attachment in sentence parsing : Evidence from dutch, The Quarterly Journal of Experimental Psycho$\log y, 49 A(3), 664-695$.

Carreiras M., Clifton C. - (1993) Relative clause interpretation preferences in Spanish and English, Language and Speech, 36 (4), 353-372.

Carreiras M., Clifton C. - (1999) Another word on parsing relative clauses : Eyetracking evidence from Spanish and English, Memory and cognition, $27(5), 826-833$.

Clifton C. - (1988) Restrictions on late closure : Appearance and reality, communication orale présentée à la $6^{\circ}$ conférence australienne « Language and Speech », University of South Wales, Sydney.

Clifton C., Ferreira F. - (1989) Ambiguity in context, Language and Cognitive Processes, $4(3 / 4)$, 77-103.

Clifton C., Frazier, L., Connine C. - (1984) Lexical expectations in sentence comprehension, Journal of Verbal Learning and Verbal Behavior, 23, 696708.

Coulson S, King J. W., Kutas M. - (1998) Expect and unexpected : Eventrelated brain response to morphosyntactic violations, Language and Cognitive Processes, 13 (1), 21-58.

Crain S., Steedman M. - (1985) On not being led up the Garden Path : The use of context by the psychological syntax processor, in D. R. Dowty, L. Karttunen et A. Zwiscky (Edit.), Natural language parsing : Psychological, computational and theoretical perspectives, Cambridge, Cambridge University Press, 320-358.

Cuetos F., Mitchell D. C. - (1988) Cross-linguistic differences in parsing : Restrictions on the use of late closure strategy in Spanish, Cognition, 30, 73105.

DeVincenzi M., Job R. - (1993) Some observations on the universality of the late closure strategy, Journal of Psycholinguistic Research, 22 (2), 189206.

DeVincenzi M., Job R. - (1995) An investigation of late-closure : The role of syntax, thematic structure, and pragmatics in initial and final interpretation, Journal of Experimental Psychology : Learning, Memory, and Cognition, 21, 1303-1321.

Ehrlich K., Fernandez E., Fodor J. D., Stenshoel E., Vinereanu M. - (1999) Low attachment of relative clauses : New data from Swedish, Norwegian and Romanian, communication écrite présentée à la $12^{e}$ conférence annuelle CUNY sur le traitement humain des phrases, cUNY, New York. 
Fernandez E., Bradley D. - (1999) Length effects in the attachment of relative clause in English, communication écrite présentée à la $12^{\circ}$ conférence annuelle CUNY sur le traitement humain des phrases, CUNY, New York.

Ferreira F., Henderson J. M. - (1990) Use of verb information in syntactic parsing : Evidence from eye movements and word-by-word self-paced reading, Journal of Experimental Psychology : Learning, Memory, and Cognition, $16(4), 555-568$.

Fodor J. A. - (1983) The modularity of mind, Cambridge (MA), MIT Press.

Fodor J. D. - (1998) Learning to parse ?, Journal of Psycholinguistic Research, 27 (2), 285-319.

Ford M., Bresnan J. W., Kaplan R. M. - (1982) A competence based theory of syntactic closure, in J. W. Bresnan (Edit.), The mental representation of grammatical relations, Cambridge (MA), MIT Press, 727-796.

Frazier L. - (1979) On comprehending sentences : Syntactic parsing strategies, thèse, University of Connecticut, Indiana University Linguistics Club.

Frazier L. - (1987 a) Theories of sentence processing, in J. L. Garfield (Edit.), Modularity in knowledge representation and natural language understanding, Cambridge (MA), MIT Press, 291-307.

Frazier L. - (1987 b) Sentence processing : A tutorial review, in M. Coltheart (Edit.), Attention and performance XII : The psychology of reading, Hillsdale (NJ), Lawrence Erlbaum, 559-585.

Frazier L. - (1989) Against lexical generation of syntax, in W. D. MarslenWilson (Edit.), Lexical representation and process, Cambridge (MA), MIT Press, 409-433.

Frazier L. - (1990 a) Exploring the architecture of the language processing system, in G. T. Altmann (Edit.), Cognitive models of speech processing: Psycholinguistic and computational perspectives, Cambridge (MA), MIT Press, 409-433.

Frazier L. - $(1990 b)$ Parsing modifiers, Special purpose routines in the human sentence processing mechanism ?, in D. A. Balota, G. B. Flores d'Arcais et K. Rayner (Edit.), Comprehension Processes in Reading, Hillsdale (NJ), Lawrence Erlbaum, 303-329.

Frazier L., Clifton C. - (1996) Construal, Cambridge (MA), MIT Press.

Frazier L., Fodor J. D. - (1978) The sausage machine : A new two-stage parsing model, Cognition, 6, 291-325.

Frazier L., Rayner K. - (1982) Making and correcting errors during sentence comprehension : Eye movements in the analysis of structurally ambiguous sentences, Cognitive Psychology, 14, 178-210.

Frenck-Mestre C., Pynte J. - (1999) Resolving syntactic ambiguities : Crosslinguistic differences?, in V. Lombardo et M. DeVincenzi (Edit.), Crosslinguistic perspectives on language processing, Netherlands, Kluwer Academic Press, 119-148.

Frenck-Mestre C., Pynte J. - (2000) Romancing syntactic ambiguity: Why the French and the Italians don't see eye to eye, in A. Kennedy, R. Radach, D. Heller et J. Pynte (Edit.), Reading as a perceptual process, Oxford, Elsevier, 549-564.

Gibson E., Pearlmutter N., Canseco-Gonzalez E., Hickok G. - (1996) Recency preference in the human sentence processing mechanism, Cognition, 59, 2359.

Gibson E., Pearlmutter N. J., Torrens V. - (1999) Recency and lexical preferences in Spanish, Memory and cognition, 27 (4), 603-611.

Gilboy E., Sopena J. M. - (1996) Segmentation effects in the processing of complex NPS with relative clauses, in M. Carreiras, J. E. Garcia-Albea, 
N. Sebastian-Galles (Edit.). Language processing in Spanish, Mahwah (NJ), Laurence Erlbaum, 191-206.

Gilboy E., Sopena J. M., Clifton C., Frazier L. - (1995) Argument structure and association preferences in Spanish and English complex NPS, Cognition, $54,131-167$.

Grice H. P. - (1975) Logic and conversation, in P. Cole et J. Morgan (Edit.), Syntax and semantic, vol. 3 : Speech acts, New York, Academic Press, 41-58.

Hagoort P., Brown C. - (1994) Brain responses to lexical ambiguity resolution and parsing, in C. Clifton, L. Frazier et K. Rayner (Edit.), Perspectives on sentence processing, Hillsdale (NJ), Lawrence Erlbaum, 45-80.

Hagoort P., Brown C., Groothusen J. - (1993) The syntactic positive shift (SPS) as an ERP measure of syntactic processing, Language and Cognitive Processes, 8 (4), 439-483.

Hahne A., Friederici A. D. - (1999) Electrophysiological evidence for two steps in syntactic analysis : Early automatic and late controlled processes, Journal of Cognitive Neuroscience, 11, 194-205.

Hemforth B., Konieczny L., Scheepers C. - $(2000 a)$ Modifier attachment : Relative clauses and coordinations, in B. Hemforth et L. Konieczny (Edit.), Sentence processing in German, Netherlands, Kluwer Academic Press, 159-183.

Hemforth B., Konieczny L., Scheepers C. - (2000 b) Syntactic attachment and anaphor resolution: The two sides of relative clause attachment, in M. Crocker, M. Pickering et C. Clifton (Edit.), Architecture and mechanisms of language processing, Cambridge, Cambridge University Press.

Hemforth B., Konieczny L., Scheepers C., Strube G. - (1998) Syntactic ambiguity resolution in German, in D. Hillert (Edit.), Sentence comprehension : A cross-linguistic perspective, New York, Academic Press, 293-312.

Henstra J. - (1996) Relative clause attachment in English : Eye-tracking versus self paced reading, communication orale présentée à la conférence « Architectures and Mechanisms for Language Processing », Turin, Italie.

Igoa J. M., Carreiras M., Meseguer E. - (1998) A study on late closure in Spanish : Principle-grounded vs. frequency-based accounts of attachment preferences, The Quarterly Journal of Experimental Psychology, 51 A (3), 561592.

Kamide Y., Mitchell D. C. - (1997) Relative clause attachment : Nondeterminism in Japanese parsing, Journal of Psycholinguistic Research, 26, 247-254.

Kamide Y., Mitchell D. C. - (1999) Incremental Pre-head attachment in Japanese parsing, Language and Cognitive Processes, 14, 631-662.

Kennedy A., Murray W. S., Jennings F., Reid C. - (1989) Parsing complements : Comments on the generality of the principle of minimal attachment, Language and Cognitive Processes, 4 (3/4), 51-76.

Kimball J. - (1973) Seven principles of surface structure parsing in natural language, Cognition, 2 (1), 15-47.

Konieczny L., Hemforth B., Scheepers C., Strube G. - (1997) The role of lexical head in parsing : Evidence from German, Language and Cognitive Processes, $12(2 / 3), 307-348$.

Kutas M., Van Petten C. - (1998) Event-related brain potential studies of language, in P. K. Ackles, J. R. Jennings et M. G. Coles (Edit.), Advances in psychophysiology, Greenwich, JAI Press, 138-187.

MacDonald M. C., Pearlmutter N. J., Seidenberg M. S. - (1994a) Lexical nature of syntactic ambiguity resolution, Psychological Review, 101 (4), 676-703. 
MacDonald M. C., Pearlmutter N. J, Seidenberg M. S. - (1994 b) Syntactic ambiguity resolution as lexical ambiguity resolution, in C. Clifton, L. Frazier et K. Rayner (Edit.), Perspectives on sentence processing, Hillsdale (NJ), Lawrence Erlbaum, 123-153.

Marcus M., Hindle D. - (1990) Description theory and intonation boundaries, in G. T. Altmann (Edit.), Cognitive models of speech processing, psycholinguistic and computational perspectives, Cambridge (MA), MIT Press, 483512 .

Marcus M., Hindle D., Fleck M. M. - (1983) D-theory : Talking about talking about trees, communication publiée dans les actes de la $21^{e}$ rencontre annuelle de l'Association pour la linguistique computationnelle, Cambridge, Massachusetts.

Mitchell D. C. - (1989) Verb guidance and other lexical effects in parsing, Language and Cognitive Processes, 4 (3/4), 123-154.

Mitchell D. C., Brysbaert M. - (1998) Challenge to recent theories of crosslinguistic variation in parsing : Evidence from Dutch, in D. Hillert (Edit.), Sentence comprehension : A cross-linguistic perspective, New York, Academic Press, 313-335.

Mitchell D. C., Brysbaert M., Grondelaers S., Swanepoel P. - (2000) Modifier attachment in Dutch : Testing aspects of Construal theory, in A. Kennedy, R. Radach, D. Heller et J. Pynte (Edit.), Reading as a perceptual process, Oxford, Elsevier, 493-517.

Mitchell D. C., Cuetos F., Corley M. M. - (1992) Statistical versus linguistic determinants of parsing bias : Cross-linguistic evidence, communication orale présentée à la $5^{\circ}$ conférence annuelle $\mathrm{CUNY}$ sur le traitement humain des phrases, CUNY, New York.

Mitchell D. C., Cuetos F., Corley M. M., Brysbaert M. - (1995) Exposurebased models of human parsing : Evidence for the use of coarse-grained (nonlexical) statistical records, Journal of Psycholinguistic Research, 24 (6), 469-488.

Mitchell D. C., Holmes V. M. - (1985) The role of specific information about the verb in parsing sentences with local structural ambiguity, Journal of Memory and Language, 24, 542-559.

Miyamoto E. T. - (1999) Relative clause processing in Brazilian Portuguese and Japanese, thèse, Massachusetts Institute of Technology.

Osterhout L. - (1994) Event-related brain potentials as tools for comprehending language comprehension, in C. Clifton, L. Frazier et K. Rayner (Edit.), Perspectives on sentence processing, Hillsdale (NJ), Lawrence Erlbaum, 15-43.

Osterhout L., Holcomb P. J. - (1993) Event-related potentials and syntactic anomaly : Evidence of anomaly detection during the perception of continuous speech, Language and Cognitive Processes, 8 (4), 413-437.

Perfetti C. A. - (1990) The co-operative language processors: Semantic influences in an autonomous syntax, in D. A. Balota, G. B. Flores d'Arcais et K. Rayner (Edit.), Comprehension processes in reading, Hillsdale (NJ), Lawrence Erlbaum, 205-229.

Pritchett B. L. - (1988) Garden Path phenomena and the grammatical basis of language processing, Language, 64, 539-576.

Pynte J., Colonna S. - (1998) French readers sometimes prefer to attach low, communication orale présentée à la conférence « Architectures and Mechanisms for Language Processing », Freiburg, Allemagne, 24-26 septembre.

Pynte J., Colonna S. - (2000) Decoupling syntactic parsing from visual inspection: The case of relative clause attachment in French, in A. Kennedy, 
R. Radach, D. Heller et J. Pynte (Edit.), Reading as a perceptual process, Oxford, Elsevier, 529-547.

Rayner K., Carlson M., Frazier L. - (1983) The interaction of syntax and semantic during sentence processing: Eye movements in the analysis of semantically biased sentences, Journal of Verbal Learning and Verbal Behavior, $22,358-374$.

Schelstraete M. A. - (1993) La conception du traitement syntaxique en compréhension de phrases, L'Année Psychologique, 93, 543-582.

Sekerina I. - (sous presse) The late closure principle in processing of ambiguous Russian sentences, à paraître dans les actes de la $2^{\mathrm{e}}$ Conférence européenne sur la description formelle des langues slaves, Potsdam, Allemagne.

Shapiro L. P., Nagel H. N., Levine B. A. - (1993) Preference for a verb's complements and their use in sentence processing, Journal of Memory and Language, 32, 96-114.

Smith C. S., Meier R. P., Foss D. J. - (1991) Information and decision making in parsing, in C. Smith (Edit.), Current issues in natural language processing, Austin, University of Texas, Center for Cognitive Science, 1-43.

Sturt P., Crocker M. W. - (1996) Monotonic syntactic processing : A crosslinguistic study of attachment and reanalysis, Language and Cognitive Processes, $11(5), 449-494$.

Tabor W., Juliano C., Tanenhaus M. K. - (1997) Parsing in a dynamical system : An attractor-based account of the interaction of lexical and structural constraints in sentence processing, Language and Cognitive Processes, $12(2), 211-271$.

Tanenhaus M. K., Carlson G., Trueswell J. C. - (1989) The role of thematic structures in interpretation and parsing, Language and Cognitive Processes, $4(3 / 4), 211-334$.

Traxler M. J., Pickering M. J., Clifton C. - (1998) Adjunct attachment is not a form of lexical ambiguity resolution, Journal of Memory and Language, 39, 558-592.

Trueswell J. C. - (1996) The role of lexical frequency in syntactic ambiguity resolution, Journal of Memory and Language, 35, 566-585.

Trueswell J. G., Kim A. E. - (1998) How to prune a Garden Path by nipping it in the bud : Fast priming of verb argument structure, Journal of Memory and Language, 39, 102-123.

Trueswell J. C., Tanenhaus M. K. - (1994) Toward a lexicalist framework for constraint-based syntactic ambiguity resolution, in C. Clifton, L. Frazier et K. Rayner (Edit.), Perspectives on sentence processing, Hillsdale (NJ), Lawrence Erlbaum, 155-179.

Trueswell J. C., Tanenhaus M. K., Garnsey S. M. - (1994) Semantic influences on parsing: Use of thematic role information in syntactic ambiguity resolution, Journal of Memory and Language, 33, 285-318.

Trueswell J. C., Tanenhaus M. K., Kello C. - (1993) Verb-specific constraints in sentence processing: Separating effects of lexical preference from Garden Path, Journal of Experimental Psychology: Learning, Memory, and Cognition, 19 (3), 528-553.

Weinberg A. - (1993) Parameters in the theory of sentence processing : Minimal commitment theory goes east, Journal of Psycholinguistic Research, $22(3), 339-363$.

Wijnen F. - (1998) Dutch relative clause attachment in two and three site contexts, communication écrite présentée à la $11^{\mathrm{e}}$ conférence annuelle cUNY sur le traitement humain des phrases, États-Unis (NJ), New Brunswick.

Wijnen F., Troost C., Quené H. - (1999) Prosodic phrasing and relative clause 
attachment in a three site context, communication écrite présentée à la $12^{\circ}$ conférence annuelle CUNY sur le traitement humain des phrases, New York.

Zagar D. - (1988) L'utilisation du temps d'exposition comme indicateur du temps de traitement pendant la lecture, in C. Bastien, J. P. Caverni, P. Mendelsohn et G. Tiberghien (Edit.), Psychologie cognitive : modèles et méthodes, Grenoble, PUG, 293-328.

Zagar D., Pynte J., Rativeau S. - (1997) Evidence for early closure attachment on first pass reading times in French, The Quarterly Journal of Experimental Psychology, 50A (2), 421-438.

(Accepté le 31 mai 2001.) 
